\title{
A Frequency-Modulated Continuous Wave-Based Boundary Detection System for Determination of Monitoring Region for an Indoor Ultra-Wideband Short Range Radar-Based Eldercare Monitoring System
}

\author{
A Thesis \\ presented to \\ the Faculty of California Polytechnic State University, \\ San Luis Obispo
}

In Partial Fulfillment of the Requirements for the Degree Master of Science in Electrical Engineering

by Wilson Tang

June 2011 
(C) 2011

Wilson Tang

All rights reserved 


\section{Committee Membership}

TITLE:

AUTHOR:

DATE SUBMITTED:

COMMITTEE CHAIR:

COMMITTEE MEMBER:

COMMITTEE MEMBER:
A Frequency-Modulated Continuous WaveBased Boundary Detection System for Determination of Monitoring Region for an Indoor Ultra-Wideband Short Range Radar-Based Eldercare Monitoring System

Wilson Tang

June 10, 2011

Tina Smilkstein

Assistant Professor

Dennis Derickson

Department Chair

James G. Harris

Professor Emeritus

iii 


\section{Abstract}

A Frequency-Modulated Continuous Wave-Based Boundary Detection System for Determination of Monitoring Region for an Indoor Ultra-Wideband Short Range Radar-Based Eldercare Monitoring System

\section{Wilson Tang}

Falls are a cause of concern for the elderly since it can render a person immobile. A monitoring system can summon immediate aid by the automatic detection of fall events. The application of ultra-wideband (UWB) signals for a monitoring system was chosen due to its unique characteristics such as wide spectrum, immunity to interference, penetrability, and application for the detection of people. Part of this monitoring system is boundary detection with the purpose of isolating the location

of fall events and to function as a mask to define an area of interest. A study of various localizing methods using measurements such as power, distance, and angle with ultra-wideband is presented.

A frequency modulated continuous wave (FMCW) based system is an acceptable solution for boundary detection. A FMCW system measures distance with UWB signals. With the addition of a unique reflector, the system is able to identify the 
reflector via the return frequency-spectrum fingerprint. With distance and the addition angle measurements, the system can determine the boundary with a sensor located at a single location.

Keywords: ultra-wideband, UWB, FMCW, monitoring system, boundary detection, falls, reflector 


\section{Acknowledgments}

I would like to thank my advisors for the thesis idea and introducing me to the world of ultra-wideband and supporting me along each step in the process of developing this thesis. I would like to extend my gratitude to many professors who listened to the idea and gave feedback, suggestions, which caused me to pause and think.

I also appreciate the help and time from my thesis committee in finalizing this document. 


\section{Contents}

List of Tables ix

List of Figures $\quad$ x

1 Introduction 1

1.1 Monitoring System Outline . . . . . . . . . . . . . . 2

1.2 Monitoring System Requirements . . . . . . . . . . . . . . . . . 3

1.3 Previous Works on Monitoring Systems . . . . . . . . . . . . . . . . . 4

1.4 Problem Definition for an UWB-based Monitoring System . . . . . . 8

2 Ultra-wideband Background 12

$2.1 \quad$ Brief History $\ldots \ldots \ldots \ldots \ldots \ldots$

2.2 FCC Regulation . . . . . . . . . . . . . . . . . . . . 13

2.3 Signal Characteristics . . . . . . . . . . . . . . . 16

3 Boundary Detection System Background 19

3.1 Overview of Positioning Techniques . . . . . . . . . . . . . 19

3.2 Antenna Considerations . . . . . . . . . . . . . . 22 
3.3 Positioning Systems Analysis . . . . . . . . . . . . . . . 23

4 FMCW System 36

$4.1 \quad$ Component Discussion $\ldots \ldots \ldots \ldots$

4.2 Test Setup . . . . . . . . . . . . . . . . . . . . . 43

4.3 Data Discussion . . . . . . . . . . . . . . . . . . . . . 46

4.4 Ordinary Least Square Model . . . . . . . . . . . . . . . . . . . 53

4.5 Identification $\ldots \ldots \ldots \ldots \ldots \ldots \ldots$

$\begin{array}{llr}5 & \text { Conclusion } & 58\end{array}$

$5.1 \quad$ Future Work $\ldots \ldots \ldots \ldots$. . . . . . . . . . . . . . . . . 59

\begin{tabular}{lr}
\hline Bibliography & 62
\end{tabular}

\begin{tabular}{ll}
\hline A Code & 67
\end{tabular}

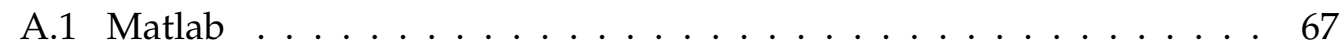

A.2 Gnuplot . . . . . . . . . . . . . . . . . . 70

\begin{tabular}{ll}
\hline Bill of Materials & 71
\end{tabular}

\begin{tabular}{ll}
\hline C Test Procedure & 73
\end{tabular}

C.1 S-parameters . . . . . . . . . . . . . . . . 73

C.2 Distance Measurements . . . . . . . . . . . . . . . . . 75 


\section{List of Tables}

1.1 Comparison of various sensor types showing UWB's strengths . . . . . . 6

B.1 Bill of materials $\ldots \ldots \ldots \ldots \ldots \ldots \ldots \ldots$ 


\section{List of Figures}

1.1 Various boundaries can be generated from a set of vertices . . . . . . 10

1.2 Positioning techniques $\ldots \ldots \ldots \ldots \ldots \ldots$

2.1 FCC mask for power limits on UWB emissions . . . . . . . . . . . . . 14

2.2 Two different transmission methods for meeting FCC requirements . . . 15

2.3 Spectral power plot of a narrowband and a UWB signal . . . . . . . . . . 16

2.4 Time domain view of a narrowband and a pulsed UWB-IR signal . . . . 17

3.1 Determining angle of arrival $(\theta)$ with an antenna array $\ldots . . . . .22$

3.2 Directional finding antenna system . . . . . . . . . . . . . . 24

3.3 Small antenna loop on $x z$-plane and element pattern . . . . . . . . . . . 24

3.4 Small antenna loop on yz-plane and element pattern . . . . . . . . . . . 25

3.5 Defining the coordinate system and $\phi$ in the smart antenna system . . . 25

3.6 Signal phase determines the quadrant . . . . . . . . . . . . . . . 25

3.7 Monopulse individual element pattern and normalized function . . . . . 27

3.8 Monopulse based four antenna element setup for omni-directional sensing 27

3.9 Monopulse positioning system block diagram . . . . . . . . . . . . 28 
3.10 FMCW system block diagram by Waldmann, et. al. . . . . . . . . . . . . 29

3.11 Frequency difference between a local signal and a received signal . . . . 30

3.12 Simulink model for a FMCW system . . . . . . . . . . . . . . . . . . 31

3.13 Time domain view of a chirp signal . . . . . . . . . . . . . . . 32

3.14 Square pulse as control signal to a switch $\ldots \ldots . \ldots . \ldots 33$

3.15 FMCW pulses . . . . . . . . . . . . . . . . . 33

3.16 FMCW output IF frequency $\ldots \ldots \ldots \ldots$

$4.1 \quad$ Block diagram for system test setup $\ldots \ldots \ldots$. . . . . . . . . 37

4.2 Various test setups used for characterizing the FMCW system . . . . . 38

4.3 Schematic of bi-directional coupler . . . . . . . . . . . . . . . 39

$4.4 S$-parameters for the bi-directional coupler $2.7 \mathrm{GHz}$ to $5.0 \mathrm{GHz}$. . . . . . 40

4.5 Mixer insertion loss with LO port power of $+10 \mathrm{dBm} \ldots . . . . . .44$

$4.6 \quad S$-parameters for LNA $\ldots \ldots \ldots$. . . . . . . . . . . . . . 42

4.7 Front view of test setup for distance measurements with a reflector . . . 44

4.8 Back view of test setup for distance measurements with a reflector _ . . 44

4.9 Test board containing the mixer and bi-directional coupler . . . . . . . 45

4.10 Two stage amplifier connected between the receive antenna and mixer . 46

4.11 Oscilloscope output for a coaxial cable length of $120 \mathrm{~cm}$. . . . . . . . . 47

4.12 Oscilloscope output for a distance $272 \mathrm{~cm}$ through air . . . . . . . . . . 48

4.13 Oscilloscope output for a distance $528 \mathrm{~cm}$ in air with a reflection . . . . . 48

4.14 Spectrum of five samples for a cable length of $120 \mathrm{~cm} \ldots$. . . . . . . . 49

4.15 Spectrum of five samples of a distance $528 \mathrm{~cm}$ in air with a reflection . . 50

4.16 Output frequency using different cable lengths . . . . . . . . . . . . 51

4.17 LNA circuit schematic and layout from datasheet . . . . . . . . . . 52

4.18 Output frequency with using different cables length and an amplifier . . 52

4.19 Output frequency from signal transmitted through air . . . . . . . . . 53 
4.20 Output frequency from signal transmitted through air with a reflection . 54

4.21 Plausible real environment FMCW response with unique reflector . . . . 56

4.22 Spectrum for two objects spaced apart by $25.4 \mathrm{~cm}$. . . . . . . . . . . 57

C.1 Block diagram for system test setup $\ldots \ldots \ldots \ldots \ldots$ 


\section{Introduction}

An electronic monitoring system to aid in caretaking of individuals was conceptualized by Dr. Tina Smilkstein [1]. This system allows individuals to continue to live independently without a personal caretaker. The preliminary goal for this system is an unobtrusive monitoring system for falls and other events that require immediate care or signal an inability for the individual to care for themselves. The electronic monitoring system exploits the features of ultra-wideband and uses an ultra-wideband (UWB) short-range radar (SRR) as its sensor. This thesis focuses on boundary detection, a subpart of the electronic monitoring system, which identifies the boundary of the region to be monitored. This simplifies signal processing and avoids monitoring unrelated activities. A system and corresponding circuitry to solve this issue is presented. Before discussing the boundary detection circuit details, the complete system will be introduced and the strengths of this type of system over systems presently being researched and deployed will be discussed. A frequency-modulated continuous-wave (FMCW) based system will be shown to fit the application of boundary detection. A Simulink model simulation shows the operation of the system, and a prototype is built and tested. The test results shows that the FMCW system can accurate measure out to 5 meters where the 
frequency output shows a direct linear relationship with the distance. A special reflector with a spacing of $25.4 \mathrm{~cm}$ used for its unique fingerprint. The reflector spacing was measured with an error of $0.5 \mathrm{~cm}$.

\subsection{Monitoring System Outline}

Dr. Tina Smilkstein envisioned an unobtrusive monitoring system which has a wide range of monitoring abilities in a single sensor system to monitor health. Elderly falls are an issue since once the subject has fallen, the subject may be immobile and injured such that they cannot rise from the floor. One of the purposes of this system is to detect these falls and summon aid to rescue the subject. This system is divided into four subparts with a different research group focusing on each subpart. Those parts are

boundary detection defining the region where detection should be focused

silhouette capture system techniques to process and capture a human silhouette

identification system differentiating between individuals when other individuals are in the monitoring region within a boundary

data transmission communication and the sharing of data between the sensor and a processing unit

The work in this thesis focuses on the first part. The application of boundary detection is to define the region where events should be detected. The occurrence of an event should not trigger alarms if they happen at a neighbor's location. The result of this can also be integrated into mitigating multipath signals by eliminating signals that have taken longer than a signal would, if it had come from a direct reflection from inside the boundary. The imaging system has the purpose of capturing a silhouette 
of the target. Living quarters contain many objects which can reflect RF signals or contain RF emitter sources. The identification system separates multiple individuals within a boundary.

Data transmission is to transmit data to a central processing unit that can track trends and perform analysis. A backend can be used as the control center of remote sensors and function as data processor to analyze if a fall has occurred. Having the analysis done by a reconfigurable backend instead of fixed hardware allows for additional systems to be easily added, existing analysis to be changed and analysis results to be used together to gather even more information on a subject.

The purpose of the system is to extend independent living among the elderly and those with physical or mental challenges. This allows for full-time electronic monitoring compared to having a full time caretaker or residing in a nursing home. The system can also be installed in nursing homes, as limited staff cannot simultaneously monitor all rooms at every instant.

Ultra-wideband (UWB) is used to implement this system due to the qualities of its spectrum. The total band allows for unlicensed use of $7.5 \mathrm{GHz}$ of bandwidth [2]. The pulsed nature and bandwidth of the signals also allows it to be robust against classical narrowband interference. Also, due to its frequency, the signal can also pass through thin walls with minimal attenuation. This allows for a signal transceiver to cover multiple rooms which allows for a reduction in production costs, maintenance and setup complexity. Its low power transmission does not interfere with medical equipment operation.

\subsection{Monitoring System Requirements}

The requirements for the complete monitoring system arise from the goal of helping the elderly and those with physical or mental challenges. The monitoring system 
should have low maintenance, inexpensive materials, and manageable installation cost. This allows the product to help the most people possible by lowering financial constraints. The monitoring system should be unobtrusive, such that after it is installed it does not alter the user's daily lives in a negative way. Finally, the data captured from the system must be accurate. During the design of the boundary detection subpart, the requirements of the complete monitoring system must be considered.

An unobtrusive monitoring system is one that protects the privacy of the person being monitored, does not psychologically affect the person, and is esthetically pleasing. For example, if the system is large or is unpleasing to look at or is embarrassing, people are much less likely to be willing to accept and use the system. These factors are important after deployment to ease the tension between technology and those being monitored.

The systems published and available at this time have limited functionality and clash with many of these system requirements. Noting this, research groups led by Dr. Tina Smilkstein sought to create a better system that would satisfy as many of the requirements as possible. The work determined that ultra-wideband short range radar (UWBSRR) can provide monitoring abilities beyond what presently available systems for monitoring of individuals with mental or physical challenges can offer in an unobtrusive, inexpensive, easy to setup and maintain, expandable monitoring system. UWBSRR also allows consolidation of many sensors into a single system.

\subsection{Previous Works on Monitoring Systems}

Research done by other groups with different sensors show how ultra-wideband can overcome problems that arise with other sensor types. Ultra-wideband has been used in research for applications in the medical field. A simple commercial fall alert 
system will also be introduced to show the short comings of a system currently on the market.

\section{Tracking and Fall Detection Sensors}

Investigations on monitoring systems using various sensors have been carried out, each with different requirements and specifications. Each sensor has its own unique advantages and disadvantages. Some parameters to consider are cost, line-of-sight (LOS) requirements, number of sensors, obtrusiveness, and processing complexity.

Ultrasound sensors have been used for monitoring. These sensors emit a physical wave that is blocked by physical barriers such as walls, such that they require many sensors to be placed for adequate monitoring.

Video cameras can be used to track subjects, but suffer with issues regarding line of sight and blind spots such that multiple cameras are required for a monitoring system. If a person is obstructed by an object such as a blanket, then tracking the person becomes impossible. A major concern of application is privacy of the persons being monitored. Complex video and image processing to create silhouettes are needed to protect the privacy of the people being monitored in their homes [3]. Silhouettes of people used for tracking are combined with fall detection algorithms determine if someone has fallen. Infrared cameras can help for certain cases, but it does not solve the issue of lowering the number of required cameras.

Fall detection research has included using smart carpets [4]. Smart carpets are embedded with electronic sensors, such as pressure sensors, to detect the presence of a person. Installation of these sensors requires a change in the flooring of all rooms in a house.

Wearable fall detectors are sensors which contain accelerometers, and with signal processing can determine if a fall has occurred. This sensor is worn on the person's 
Table 1.1: Comparison of various sensor types showing UWB's strengths

\begin{tabular}{lcccc}
\hline Sensor & LOS Required & $\begin{array}{c}\text { Number of } \\
\text { Sensors }\end{array}$ & Obtrusive & Processing \\
\hline UWB Based & No & Few & No & Medium \\
Ultrasound & Yes & Many & No & Low \\
Video Cameras & Yes & Many & Yes & High \\
Thermal Cameras & Yes & Many & Yes & High \\
Smart Carpets & Yes & Many & No & Low \\
Wearable Sensors & No & One & Yes & Low \\
\hline
\end{tabular}

body, but people tend to remove sensors temporally, and forget that it is removed. Also, Alzheimer's patients can also have this problem, and not understand the purpose of the sensor.

Table 1.1 lists various sensor types that can be used for fall detection. Ultrawideband is a strong candidate for fall detection as it can limit the number of sensors and cost due to its ability to penetrate walls, while remaining an unobtrusive system. Ultra-wideband impulse radio (UWB-IR) was shown in [5] to be $95 \%$ accurate in discerning between the following states: sleeping in bed, sitting up in bed, falling down, wandering in room, going out and in a door.

\section{Commercial Elderly Fall Alert System}

The eldercare market has created commercial solutions for eldercare monitoring. In the United States, $\$ 309$ billion is spent annually on eldercare [6]. For "informal care" which are services to assist seniors at home, the market estimate is $\$ 102.7$ billion annually [6]. There is market potential for products to assist seniors with growth corresponding with the population of Baby Boomers. Part of this market could be spent on electronic based monitoring systems to provide services.

A commercial solution LifeAlert [7] is an in-home monitoring system for enabling those who have fallen to contact emergency services. The user wears a pendant which 
interacts with a base station that is connected to the home's landline. This system is analogous to a cordless phone handset for a home's landline service. The pendant has one button to call for help. The user is then put into contact with the services 24/7 help line, and will contact the proper authorities to summon help after determining the current emergency. This service requires the user to have the pendant with them at all times, making this system obtrusive. The user must understand how to operate the pendant which can become a problem for patients with Alzheimer's. The person must also be capable of using the pendant after they have fallen, thus they cannot be unconscious. Besides initial equipment and installation costs, the service also requires a monthly fee to support their call center, which is a maintenance cost.

There are other companies selling similar devices and services [8], including one which directly connects with family or 9-1-1 service, where no monthly service fee is required $[9,10]$.

\section{Related Applications of Ultra-wideband}

Research in ultra-wideband includes applications for tracking and monitoring humans, and their vital signs. Techniques have been developed to monitor breathing [11, 12] and heart beats [13]. In [14], human activities such as walking, running, standing, and transitioning from sitting and standing were studied. The success rate for detecting the transition between sitting and standing is 72.6\% [14]. Human imaging has been done by combining synthetic aperture radar (SAR) with UWB [15].

The impedances of various tissues in the human body are different [16, 17]. Measurement shows that the dielectric constants of tissues carry a greater difference at lower frequencies. When an electromagnetic wave meets a boundary, the wave can be absorbed, reflected, or transmitted. It is possible to use a boundary such as the air and skin boundary with the fat and muscle boundary for human identification. Human 
tissue and water boundaries can be detected for monitoring water accumulation in bladders [18]. With the addition of an ultra-wideband signal, the impedance of tissues also vary with frequency, allowing for more characteristics to be used for human identification. Another medical applications is using tissue impedances to detect cancer [19].

A study using ultra-wideband for human detection and tracking by S. Chang, et. al [20] resulted in a $80 \%$ detection probability and a $1.58 \%$ false detection with the ability to track multiple targets. The setup used multiple human targets and an aluminum foil covered basketball as a small animal. The test subjects who entered and exited the detection region of the radar were tracked. A range of $5 \mathrm{~m}$ was used. The research combined multiple algorithms and procedures to preform the tracking including CLEAN algorithm for multipath deconvolution, Multi-Hypothesis-Testing

and Kalman filtering. The technique tracks scatter components back to the original source for multiple target tracking. This requires a human reflection and scattering models to be developed for processing. Although human identification and tracking is not a topic for the thesis, the application of ultra-wideband is not only applicable for boundary detection, but is useful in other monitoring areas.

\subsection{Problem Definition for an UWB-based Monitoring System}

Boundary detection is the process of defining a region of interest to monitor for occurrences of events. This is one of the major parts of a monitoring system. The technique of determining the unique boundary is discussed, then is translated to the steps that are performed, and the measurements needed. From the measurements needed, the system requirements are developed. 


\section{Requirements}

This thesis focuses on defining a boundary as a region of operation for the monitoring system. This boundary is typically a room or an arbitrary area in a dwelling such as a nursing home. The region is used to map areas that the system should ignore and not monitor, such as a neighbor's home. Also, the issue with multi-path return signals can also be alleviated by ignoring signals which originate outside of this boundary. Another requirement of the system is to have an ability to uniquely identify the reflector placed at a corner. To solve this problem, first a precise definition of a boundary is defined, and solutions to recreate this boundary are proposed.

\section{Boundary Definition}

A boundary is modeled as a polygon with edges and vertices. If this boundary was a room, an example would be edges are walls and vertices are corners. This boundary can also be arbitrary in space marking an area. For defining a unique boundary a set of vertices and how they are connected is required. With only a set of vertices many different boundaries can be created. Figure 1.1 shows two different boundaries created from the same set of vertices, where additional area falls under the boundary, and a different section to reside outside. Although if conditions were applied to limit the choices to contain no crossing edges would shrink the combinations, there could still be multiple solutions. Knowledge of vertices and how they are connected is important for a boundary definition.

\section{Positioning}

For positioning a point on an $x y$-coordinate system, its position is needed relative to the origin, where a known detector is placed. A method to determine position is by finding the distance between the base-station and the point, and the angle 


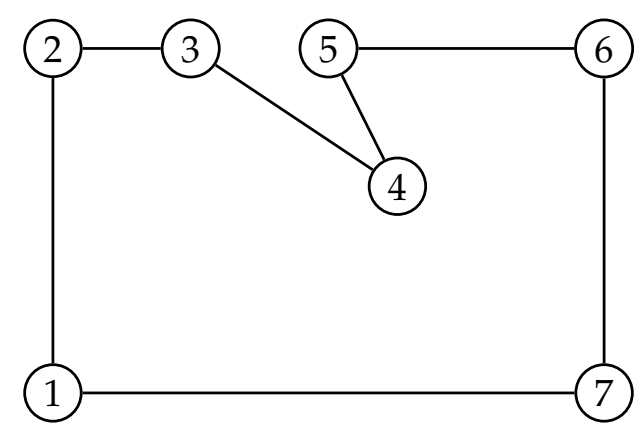

(a) correct boundary

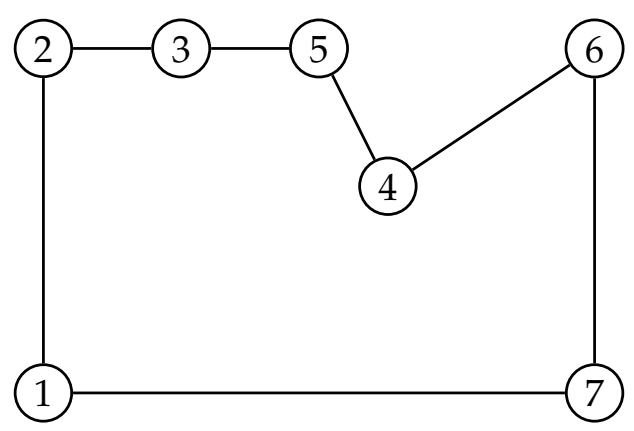

(b) incorrect variant

Figure 1.1: Various boundaries can be generated from a set of vertices

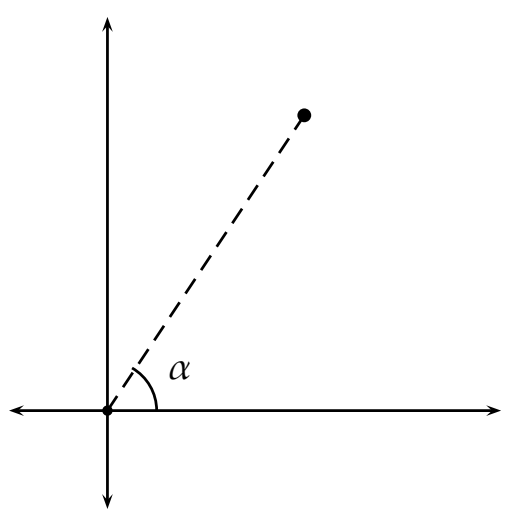

(a) Angle and distance

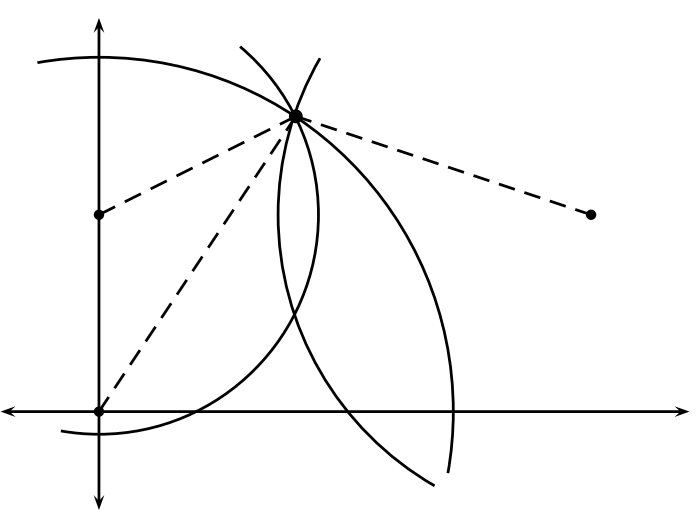

(b) Triangularization

Figure 1.2: Comparing the methods of positioning using angle and distance versus triangularization

relative to it as illustrated by Fig. 1.2(a). Another method is to use triangularization, by finding the distance from three known reference points. The position of the point is located on a circle from each reference point, and the intersection of the circles give an unique location in two-dimensions as illustrated by Fig. 1.2(b),

The advantage of having one base-station is minimal hardware setup. The installation and setup of one unit is easier. Also, the design of a method to measure distance can be applied in angle and distance or triangularization based systems. 


\section{Technique}

A proposed method to draw the room boundary is to record each vertex in order by the walls they are connected. Let vertex 1 in Fig. 1.1(a) be the reference and base-station, then a person shall place a unique sensor at each corner and allow the system to record the distance and angle. The person should repeat this for all corners, while preforming this in the proper order. This technique allows the system to create a single reflector at the first vertex. After the angle and distance are recorded by the system the reflector is moved to the second vertex. This is continued until all vertices, in order, have been recorded.

The remaining portion of this thesis discuss boundary detection with positioning by measuring distances using FMCW. In Chapter 2, an introduction to the definition and characteristics of ultra-wideband are discussed, as used for boundary detection and importance for the complete monitoring system. In Chapter 3, techniques for positioning a node are discussed, which is used to create the boundary. Chapter 4 shows the results from a FMCW based prototype which is able to measure distances. Chapter 5 contains a brief summary, concluding remarks and also discusses what lies ahead in the future. 


\section{Ultra-wideband Background}

The definition of ultra-wideband is ambiguous, such as the terms narrowband and wideband. A definition used is by comparing the ratio of signal bandwidth to its carrier frequency. At what ratio does the signal then becomes ultra-wideband? The legal definition of an ultra-wideband signal, as with all electromagnetic transmissions is regulated by the Federal Communications Commission (FCC). In this chapter, a brief history of ultra-wideband is discussed, along with the nature and characteristics of an ultra-wideband transmission.

\subsection{Brief History}

A quick history of ultra-wideband from [21], showing the evolution and its applications is summarized here, including the origin of ultra-wideband, and how it became defined and used.

In the late 1800 s, the only method to generate electromagnetic pulses was using a spark-gap generator. These pulses were short, and thus naturally wideband. This method was used by Hertz in his experiments and by Marconi for the first electromagnetic transmission. Ultra-wideband existed since the beginning. 
Later, in 1910 narrowband techniques using frequency division multiplexing was developed and techniques for efficiently using the large bandwidth were unknown. Developments using wideband signals stalled.

In the 1960s, the military was researching radar systems and by using short pulses, radar systems gained spectral resolution and accuracy. Also, the sampling oscilloscope was invented allowing for time domain analysis of the pulses. In the 1970s, the military began use of ultra-wideband for 'baseband' or 'carrier-free' communications, but spectral efficiency was not important in their application. Ultra-wideband and narrowband communications were observed to coexist without interfering with narrowband transmissions. At this point, the issue with multipleaccess interference of multiple users was not solved.

In the 1990s a time-hopping impulse radio with pseudorandom transmission times was developed to solve the multiple-access issue. Transmitters would transmit at pseudorandom times leading to a smaller chance of collisions.

In 2002, the issue with ultra-wideband was political government regulations. Traditionally, spectrum was assigned to only one user, while ultra-wideband transmissions overlapped with existing users. After many hearings, the FCC voted and addressed ultra-wideband transmissions. Standards organization such as IEEE and the WiMedia alliance developed standards for ultra-wideband transmissions. An example is IEEE 802.15.4a for low bitrate, low power data transmission with ranging built into its standard.

\subsection{FCC Regulation}

In 2002, the FCC issued its "First Report and Order" for a legal definition of ultrawideband and emission limits. The FCC allows for unlicensed ultra-wideband transmissions. The FCC definition for a UWB signal is that at any point in time it 


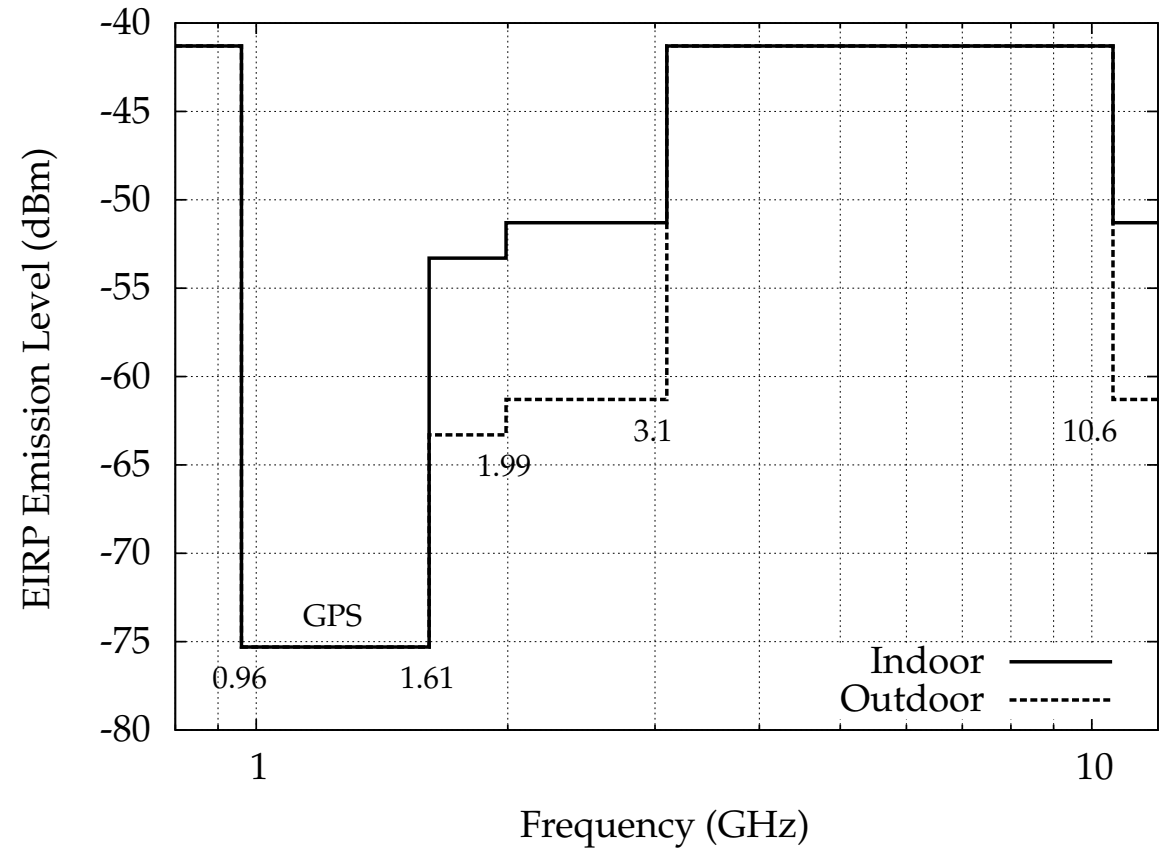

Figure 2.1: FCC mask for power limits on UWB emissions

contains $500 \mathrm{MHz}$ of spectral bandwidth. The bandwidth is measured at the points $10 \mathrm{~dB}$ below the peak power; those points are noted as $f_{L}$ and $f_{H}$. The frequency the peak power occurs at is labeled as $f_{M}$. The FCC limits emissions to an average power of $-41.3 \mathrm{~dB} / \mathrm{MHz}$, and the largest contiguous band is contained within $3.1 \mathrm{GHz}$ to 10.6 GHz as shown in Fig. 2.1. The power level of $-41.3 \mathrm{~dB} / \mathrm{MHz}$ is the same power level which the Part 15 rules allow for unintentional radiators. Bands where GPS and mobile phones are used have extra protection, below the Part 15 regulations from interference. This average power measurement is preformed with the following:

Radiated emission levels above $960 \mathrm{MHz}$ are based on RMS average measurements over a $1 \mathrm{MHz}$ resolution bandwidth. The RMS average measurement is based on the use of a spectrum analyzer with a resolution bandwidth of $1 \mathrm{MHz}$, an RMS detector, and a 1 millisecond or less 


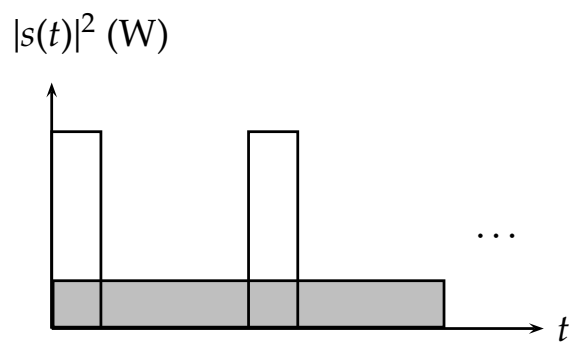

Figure 2.2: Two different power transmission methods for meeting the FCC transmission requirements

averaging time. [2]

The second power requirement on the signal is a peak power limit.

There is a limit on the peak level of the emissions contained within a 50 $\mathrm{MHz}$ bandwidth centered on the frequency at which the highest radiated emission occurs, fM. That limit is $0 \mathrm{dBm}$ EIRP. [2]

EIRP is equivalent isotropic radiated power. This is the product (or summation in terms of $\mathrm{dB}$ ) of the power supplied to the antenna and antenna gain.

$$
\operatorname{EIRP}_{\text {max }}(\mathrm{dBm})=P_{\mathrm{A}}(\mathrm{dBm})+G_{\mathrm{A}(\text { max })}(\mathrm{dB})
$$

Figure 2.2 illustrates the difference between transmitting a short, high power signal compared with a consistent lower power signal, but the average power of both cases is the same. The average power can be considered in both cases to be the same to meet regulation requirements.

Other FCC regulations include limits on various classes for indoor, imaging, vehicular radar use, or handheld devices, and how those are defined. 


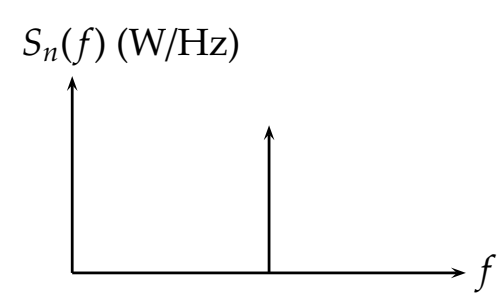

(a) Narrowband

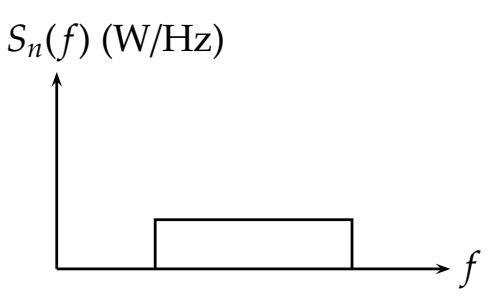

(b) UWB

Figure 2.3: Spectral power plot of a narrowband and a UWB signal

\subsection{Signal Characteristics}

Some unique characteristics arise due to the nature of ultra-wideband signals compared with narrowband signals. With a large bandwidth, the bit capacity of the channel is increased for data transmissions, although this thesis does not utilize this characteristic. The bandwidth allows for narrow pulses achieving high timeresolution [22]. Narrow pulses also help mitigate multipath concerns since two pulses must collide for interference to occur. Using various parts of the spectrum also means that multipath could be limited to a small band of the spectrum. If there is multipath due to a reflection from two paths having a half-wavelength difference in distance, then the signal only interferes for that particular wavelength. Due to using a wideband the penetration characteristics for materials also varies with frequency, allowing the signal to pass in some bands, while attenuated in others.

A plot of the spectrum of a narrowband and ultra-wideband signal is in Fig. 2.3. The power in a narrowband signal is contained within a small frequency band. If the same power of the narrowband signal is spread over a wideband, then the resulting spectral density of the signal is significantly lower.

In Fig. 2.4, the corresponding time domain view of a narrowband and ultrawideband signal is shown. The narrowband signal is a sine wave, as all its power is focused at a single frequency. The ultra-wideband signal illustrates a UWB-IR signal, 


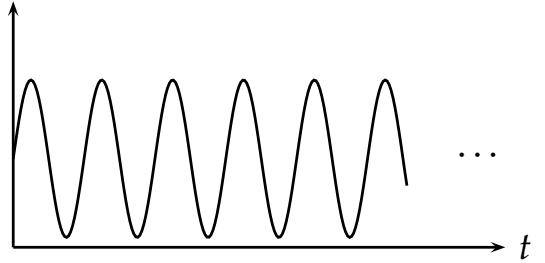

(a) Narrowband

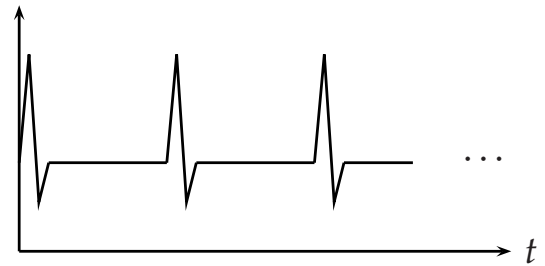

(b) UWB

Figure 2.4: Time domain view of a narrowband and a pulsed UWB-IR signal

a transmission technique using pulses, transmitted at some interval. From Fig. 2.4(b), the UWB-IR signal is a narrow pulse which corresponds to a high bandwidth signal. The two waveforms are related by their Fourier transforms. The UWB-IR signal contains a steep slope which allows it to have high time-resolution, which is the ability to discern the exact time when the signal arrives.

An example of sending higher power pulses is impulse radio, where higher power pulses are transmitted spread out over time. UWB-based orthogonal frequencydivision multiplexing (OFDM) uses multiple carriers to generate a net signal of $500 \mathrm{MHz}$, and is allowed to transmit continuously. An example is multi-band OFDM (MB-OFDM) which divides the spectrum into $528 \mathrm{MHz}$ bands within the $3.1 \mathrm{GHz}$ to 10.6 GHz band, where each band consists of 122 sub-carries [23]. After a transmission in one band is complete, it can hop into another $528 \mathrm{MHz}$ band [24]. The transmission scheme is allowed to vary the technique used to achieve their system goals.

In narrowband, multipath signals can distort the signal. A reflection can be considered as a phase offset and constructive and destructive interference can occur. In the ultra-wideband scenario, interference only can occur when two transmitters transmit at the same time, or two pulses arrive at the receiver at the same time. This allows for UWB to be more robust against multipath and interference. Experiments 
preformed by Lawrence Livermore National Labs [25] on a cargo ship illustrate this concept. The experiment transmitted a video signal from a room within the cargo ship to various other locations such as a stair well, and different floors. The walls of a cargo ship are metal, thus narrowband cannot function within the cargo ship due to interference. In a ultra-wideband system, the walls help reflect and guide the signal to the receiver for non-line-of-sight communications. 


\section{Boundary Detection System}

\section{Background}

There exists different positioning systems, but generally the positioning methods can be categorized by received power, time-based, interference based, or phase based. Typically, implementations use a hybrid of techniques to find either distance, position or both. The characteristics of the antenna used changes how the system functions and what the detector can sense. The antenna affects distance and the radiation pattern effects the region of detection. The illustration of various positioning system shows how multiple sources of data can be combined to locate a unique position in physical space. Information required for positioning are distances and angles.

\subsection{Overview of Positioning Techniques}

Various techniques of finding distances to position a node using RF signals are analyzed. For each method, a discussion of how the method functions and the data points the method requires shows the trade-offs of each technique. Next, the positioning methods are combined with ultra-wideband signals and the results of 
using an ultra-wideband signal are explored.

\section{Received Power}

A distance can be measured due to power loss from the signal traveling through space. The channel, traversed by the signal, needs to be characterized for accurate results [22]. The channel model also varies with frequency. The loss of a signal traveling through free-space is called FSPL (free-space path loss)

$$
\begin{aligned}
\mathrm{FSPL} & =\left(\frac{4 \pi d}{\lambda}\right)^{2} \\
& =\left(\frac{4 \pi d f}{c}\right)^{2}
\end{aligned}
$$

where $\lambda$ is the signal wavelength, $f$ is the signal frequency, and $d$ is the distance traveled. This model ignores the shaping effects due to antenna characteristics.

This model is only valid in free space, and is not valid if there are any multipath signals which alter the received power level. To use receive power as a method for measuring distances, a system calibration is required. If the system was relocated or moved or if the environment changes such as rearranging furniture, then a recharacterization of the channel is necessary. An ultra-wideband signal's energy is contained within a large bandwidth, thus the power received will also vary at every frequency. Besides the channel model, the frequency dependence of system components is also considered. The non-idealities such as return loss also alter the transmitted and received power. The effective power transmitted and received is also shaped by the antenna's directivity and gain.

\section{Time of Arrival}

Time of arrival (TOA) is a time based approach which uses the time of fight for electromagnetic waves, which is at the speed of light, as the means of measurement. 
A method is implementing a fast sampling circuit to measure the signal to obtain a time difference. By using short pulses, the resolution of the result is increased as the ability to finely time the arrival of the pulse is increased.

The challenge of design for this system is implementing a high sampling receiver to time when the signal is received. Clock jitter is an important system parameter in TOA techniques [26].

\section{Angle of Arrival}

The angle of arrival can be determined with an antenna array to determine the phase difference between the different antenna elements due to the incoming wavefront. Figure 3.1 shows how an antenna array can be used to determine the angle of arrival. An incoming waveform from the far field is shown, so it can be assumed to have constant phase wavefronts. With an antenna array, the distance between each antenna element is known, thus $d$ is known. The phase difference $\phi$, in radians is measured and is converted into a physical distance by

$$
d_{\phi}=\frac{\phi}{2 \pi} \lambda
$$

where $\lambda$ is the wavelength of the incoming waveform, or $c / f$. Using geometry, then resulting angle of arrival is

$$
\theta=\arccos \frac{d_{\phi}}{d}
$$

Antenna array's increase system cost, and with wide bandwidths there can be many signal paths from scattering and multipath [26].

\section{Hybrid Systems}

Systems that are currently being developed typically combine various methods together for better results or develop modifications to improve performance. 


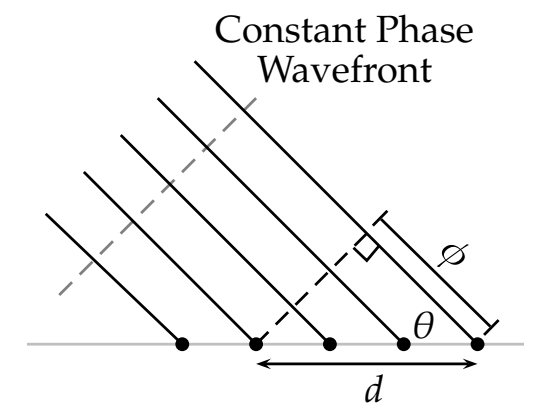

Figure 3.1: Determining angle of arrival $(\theta)$ with an antenna array

In [27], two monopole antennas, spaced apart at a known distance were used to position an active UWB source. Measurements were preformed with the antennas spaced from $10 \mathrm{~cm}$ to $50 \mathrm{~cm}$. The range tested was up to $12 \mathrm{~m}$. An oscilloscope was used to receive the two signals and processed by a computer. The computer used cross-correlation to determine the exact time of arrival of the signal. Using geometry, then the distance and angle can be found. For distance measurements, the standard deviation was $3.1 \mathrm{~mm}$. For the distance and angle measurements, the standard deviation of the positioning (Euclidean distance) error was $37.2 \mathrm{~cm}$. This system can only measure from $0^{\circ}$ to $180^{\circ}$, as it has front-back ambiguity.

\subsection{Antenna Considerations}

Factors for antenna selection in a monitoring and boundary detection system include physical size, number of antennas, and directionality. The number of antennas and physical size creates a physical constraint of the final system. The requirements of directivity and element pattern of the antenna will vary depending upon the methodology and system used to monitor or determine the boundary. A directive antenna would have a limited direction where it is sensitive to signals, while an omni-directional antenna can sense in all directions. The beamwidth of the antenna 
describes the angle which the antenna can detect signals. A trade-off can be antenna gain, where some directive antennas can sense longer distances than one with a wider beamwidth. A limited beamwidth has the advantage of allowing the system to know that the signal originated within a limited area.

The beamwidth of the antennas define constraints for the placement of the system. With an omni-directional system, the unit can be placed in the center of a room or dwelling, while with a directional system it should be placed at room corners or walls, where the antenna is facing inwards towards the living areas.

\subsection{Positioning Systems Analysis}

\section{Element Pattern}

There are techniques which require special characteristics from the antenna's radiation pattern for determining angle of arrival. One method uses two orthogonal antennas with a $\sin \theta$ and $\cos \theta$ pattern, while another is based on monopulse radar and uses a lookup table to determine angle.

\section{Orthogonal Antennas}

A technique for determining angle and distance was proposed in [28]. The technique used time of arrival as the method for determining distance, but it used a two element antenna array with a rake antenna to determine the received angle. The antenna system is shown in Fig. 3.2

The antennas used were two orthogonal loops, one is on the $x z$-plane as shown in Fig. 3.3(a), and the other on the yz-plane as shown in Fig. 3.4(a). The two loops are electrically separate, but positioned at a $90^{\circ}$ angle to each other. An antenna loop in the $x z$-plane will have a radiation pattern that is a torus located in the 


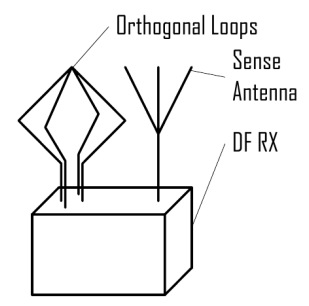

Figure 3.2: Directional finding antenna system used in proposed system in [28]

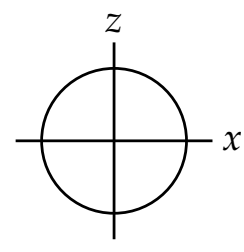

(a) Antenna
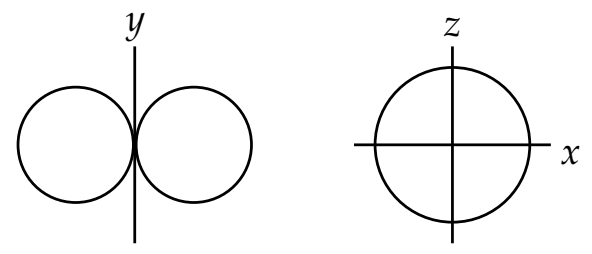

(b) $y$-plane cut element (c) $x z$-plane element pattern

Figure 3.3: Small antenna loop on $x z$-plane and element pattern

$x z$-plane (Fig. 3.3(b)) with nulls on the $z$-axis (Fig. 3.3(c)). The element pattern for an electrically small loop located on the $y z$-plane is

$$
E P_{1}=\sin \phi
$$

and the corresponding loop rotated $90^{\circ}$ on the $x y$-plane is

$$
E P_{2}=\cos \phi
$$

where $\phi$ is the angle of signal incidence referenced to the $x$-axis, as shown in Fig. 3.5 .

By considering the ratio of the received power, the resulting output follows a tangent pattern. The corresponding angle can be found by

$$
\phi=\arctan \frac{E P_{1}}{E P_{2}} .
$$

One issue which arises is the ambiguity if the signal was received in which quadrant, since there are two possible solutions of tangent. The author solves this issue by 

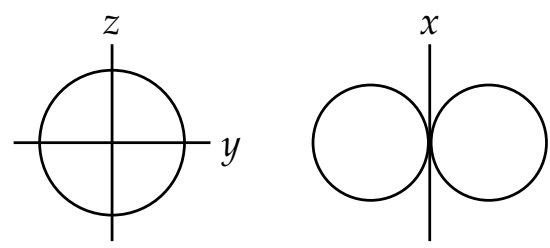

(a) Antenna (b) $x$-plane cut element (c) yz-plane element pattern

Figure 3.4: Small antenna loop on yz-plane and element pattern

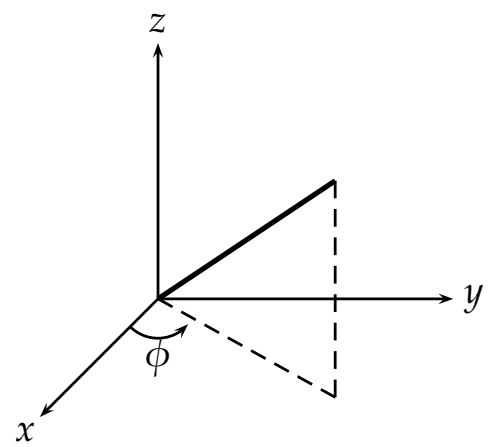

Figure 3.5: Defining the coordinate system and $\phi$ in the smart antenna system

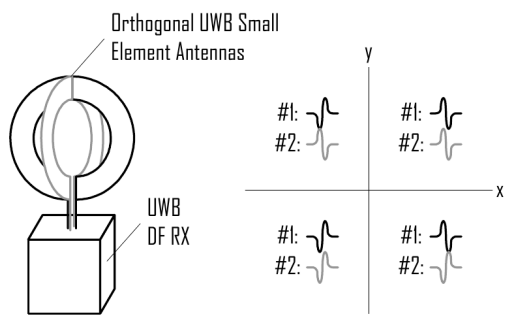

Figure 3.6: The phase of the received signal on each antenna element gives the quadrant.

considering the phase of the transmit signal. Depending on what side of the antenna the signal lands on, the signal will have a phase difference of $180^{\circ}$. This concept is illustrated in Fig. 3.6. In narrowband applications, the sense antenna is used to solve 
the front/back ambiguity, but is not necessary for UWB.

This system allows the use of one base station to make angle and distance measurements. By observing the nature of a tangent function, when the angle is near $90^{\circ}$ or $-90^{\circ}$, the function asymptotically reaches $+\infty$ and $-\infty$ respectively. If the system were to contain some finite error in measuring $E P_{1}$ and $E P_{2}$, the resulting error will increase at near the limit cases. At the nulls the received signal should be at zero, but in practice that is not achievable. Another consideration is that this method relies upon the antenna's element pattern, in this case an electrically small loop. Typically an electrically small loop has a circumference of $\lambda / 10$. For a signal at $3.1 \mathrm{GHz}$ the loop's circumference is $9.67 \mathrm{~mm}$, and for a signal at $10.6 \mathrm{GHz}$ the circumference would be $2.83 \mathrm{~mm}$. The efficiency of this antenna would also be low due to its size and would gather less RF energy from the air. Other loop type antennas can be used, but it is difficult to discover one with an ideal pattern.

\section{Monopulse Radar}

Monopulse radar was invented by Robert Page in 1943 [29]. This radar technique uses multiple antennas. A modified version designed to work with ultra-wideband was proposed in [30]. In this system, antenna elements which contain one main lobe are selected for this application. Each element is arranged in a circle such that the net antenna will be able to sense in $360^{\circ}$. As an example shown in Fig. 3.7, if the main lobe of the selected antenna element was $45^{\circ}$, then four antennas would be required.

The resulting radiation pattern of the antenna array is in Fig. 3.7. The main lobes of the antenna are located $90^{\circ}$ apart. At any angle, the received signal power will excite two antennas. Let the power received on antenna one be $g_{1}(\phi)$ and the power on antenna two be $g_{2}(\phi)$. Monopulse radar uses a normalize difference to determine 


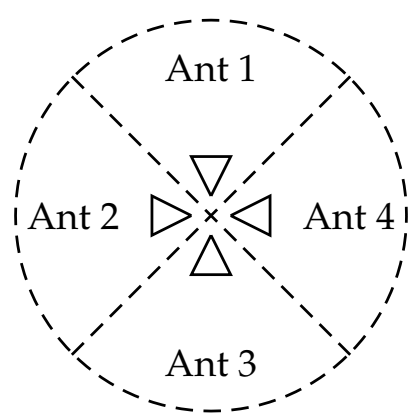

Figure 3.7: Monopulse individual element pattern and normalized function

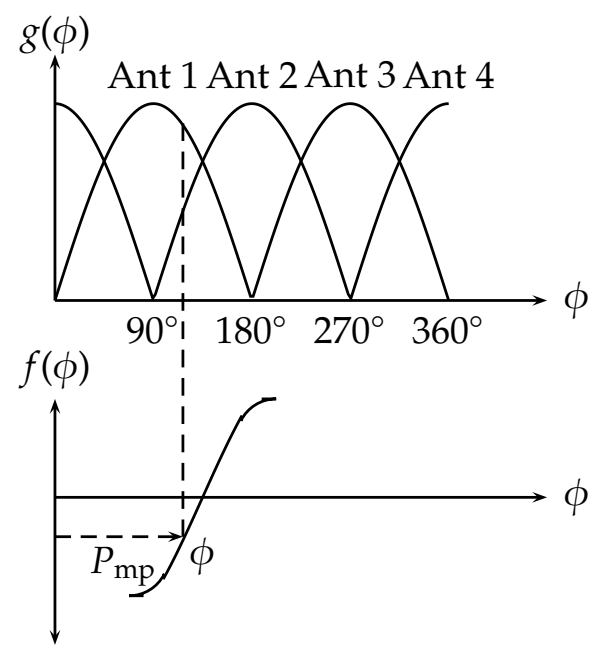

Figure 3.8: Monopulse based four antenna element setup for omni-directional sensing 3.9

the angle given by

$$
f(\phi)=\frac{g_{2}(\phi)-g_{1}(\phi)}{g_{2}(\phi)+g_{1}(\phi)}
$$

The summation factor of $g_{2}(\phi)+g_{1}(\phi)$ is used for normalization. The authors implemented this technique using a lookup table. First, a calibration is preformed which is used to generate the lookup table. Subsequent measurements are then compared with values in the lookup table. The stability of the circuit over three dates were found to be stable, such that the standard deviation of the measurement 


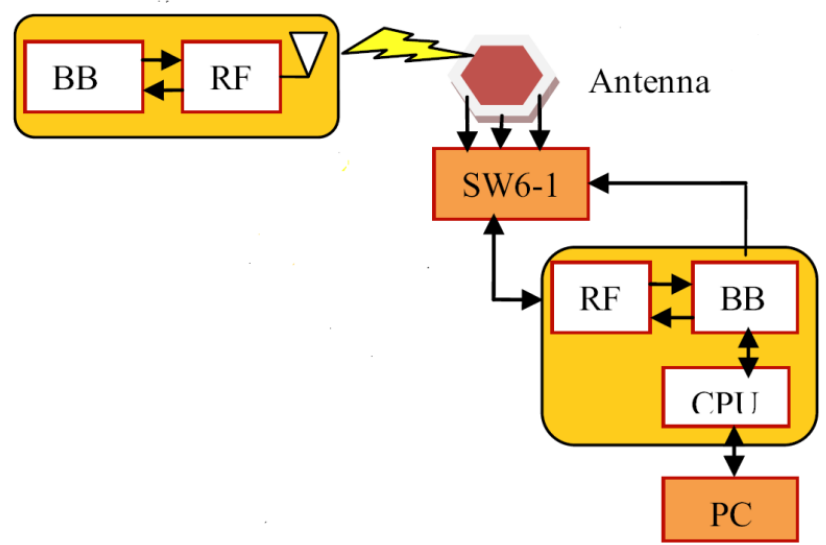

Figure 3.9: Monopulse positioning system block diagram [30]

is less than $1^{\circ}$.

The authors implemented and tested this system and included some features to lower the system cost. The system developed is in Fig. 3.9. A computer is used to control the antenna array. A six to one switch for a six element antenna array is used such that the base station only requires one RF frontend instead of one per antenna. A remote, electrically powered, active tag is used which responds to the base station's commands. With only one RF frontend at the receiver, the active tag transmits its signal six times, once per each antenna, so that energy levels can be measured individually for each antenna. In addition a coding system to allow for multiple tags and data transmission was also included in the design. The experimental results found that the system can be used up to a distance of $10 \mathrm{~m}$ with $2 \mathrm{~cm}$ ranging error, less than $1^{\circ}$ error in angle, and a raw data rate of $12 \mathrm{kbps}$. The system supports an update rate of $50 \mathrm{~Hz}$ which is adequate for human speed applications.

The unique feature of this system is that a relatively small antenna array with elements placed close to each other is adequate for measurement compared with a traditional localization method of using three nodes for positioning. 


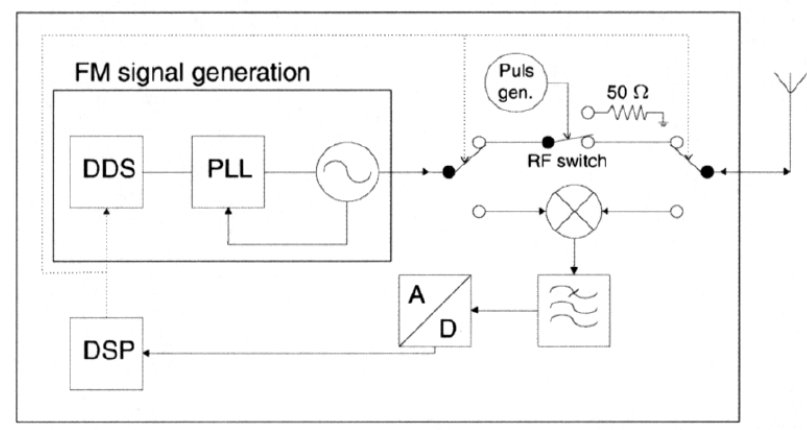

Figure 3.10: FMCW system block diagram proposed in [31]

\section{Frequency Modulated Continuous-Wave (FMCW) Radar}

A proposed FMCW system in [31] is shown in Fig. 3.10. The system is a frequency modulated continuous wave (FMCW) based radar system with ultra-wideband support. A linear FMCW source is generated with a direct digital synthesizer (DDS) as a frequency reference to a phased-locked loop (PLL) with a wideband voltage controlled oscillator (VCO). The output is connected to a RF switch to pulse the signal, at a slow rate relative to the frequency of the FMCW source. The received signal is mixed with the local signal, sampled, and processed to determine the distance.

The principle of FMCW works upon sensing the frequency difference at the mixer. The local FMCW source sweeps at a known rate. When the signal is returned its frequency will be different due to the time that has past. As shown in Fig. 3.11, the signal to the left is the local signal, while the signal to the right is the received signal. With this frequency difference, the time difference and thus distance can be determined.

The constant $k_{f}$ is defined as

$$
k_{f}=\frac{B W}{T_{\text {sweep }}}
$$




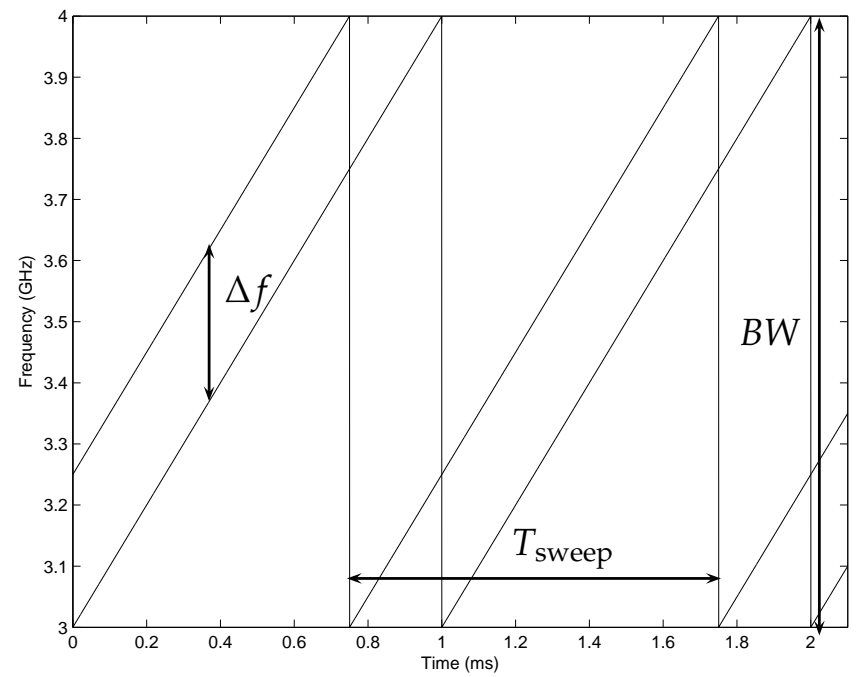

Figure 3.11: Frequency difference between a local signal and a received signal

where $B W$ is the sweep bandwidth and $T_{\text {sweep }}$ is the sweep period. This describes the slope of the sweep, and is used to determine the time difference with the measured frequency difference,

$$
t=\frac{\Delta f}{k_{f}}
$$

Time and distance can be related by the signal preparation velocity, typically through air,

$$
d=v_{p} t
$$

Combing the results of (3.6) and (3.7) results in

$$
d=\frac{v_{p} \Delta f}{k_{f}}
$$

The propagation velocity is $c$ for air and $0.7 c$ for RG-142 coaxial cable.

The range resolution of a FMCW system is given by

$$
\Delta R=\frac{c}{2 \cdot B W}
$$




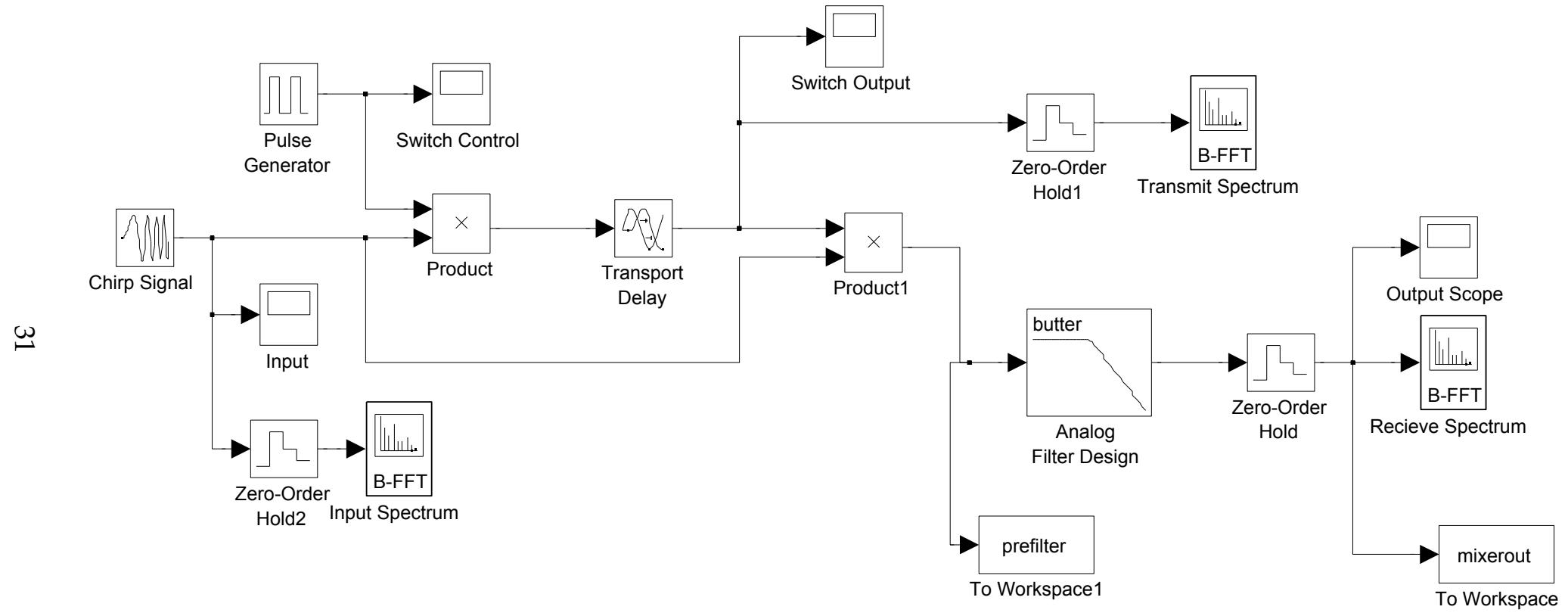

Figure 3.12: Simulink model for a FMCW system 


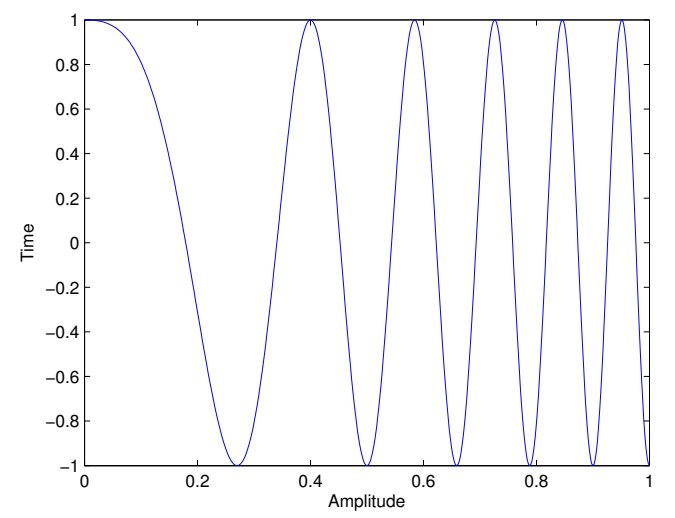

Figure 3.13: Time domain view of a chirp signal

The range resolution is the radar's ability to discern two objects placed at a distance $\Delta R$ apart. The range resolution of the radar system is solely determined by the systems sweep bandwidth $(B W)$.

The FMCW system was simulated in Simulink, as shown in Fig. 3.12. The frequency output of the Chirp Signal was in Fig. 3.11, an exaggerated time domain view is in Fig. 3.13 .

Mathematically, for one sweep period

$$
\begin{aligned}
f(t) & =\cos \left(2 \pi\left(f_{0}+k_{f} t\right) t\right) \\
& =\cos \left(2 \pi\left(f_{0} t+k_{f} t^{2}\right)\right) .
\end{aligned}
$$

The transmitter block of the FMCW system is simulated with the three Simulink blocks. The Pulse Generator block creates a square wave signal functioning as a switch control signal. The switch contains two parameters an switch on time $T_{\text {on }}$ relative to it's pulse period or $T_{\text {period }}$ as shown in Fig. 3.14. The resulting signal is inputted into a Product block, to simulate a switch, as shown in Fig. 3.15. The output of the product block follows the chirp signal when the pulse is a ' 1 ' and when the pulse output is ' 0 ', then the product output is ' 0 '. 


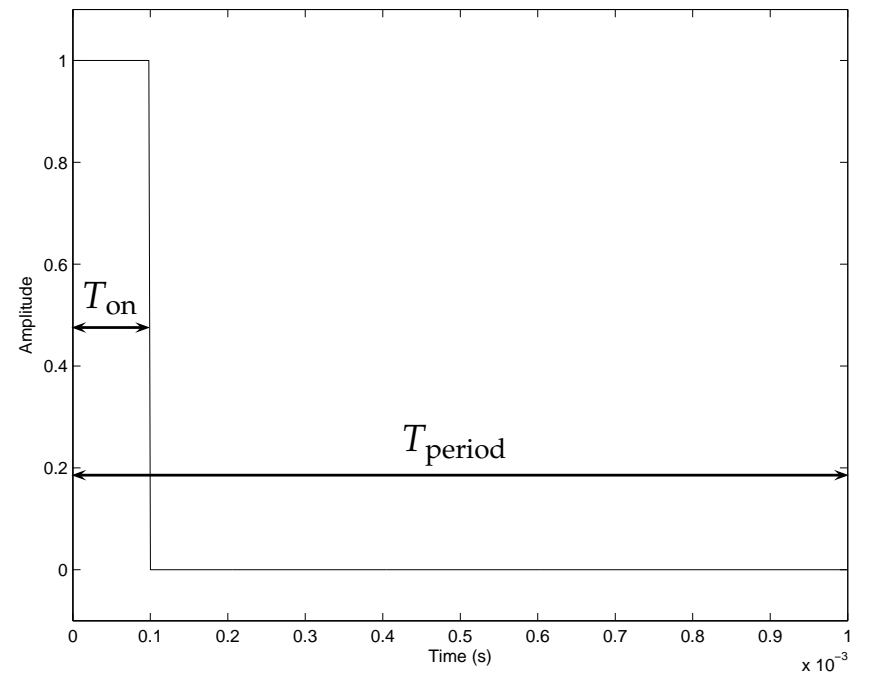

Figure 3.14: Square pulse as control signal to a switch

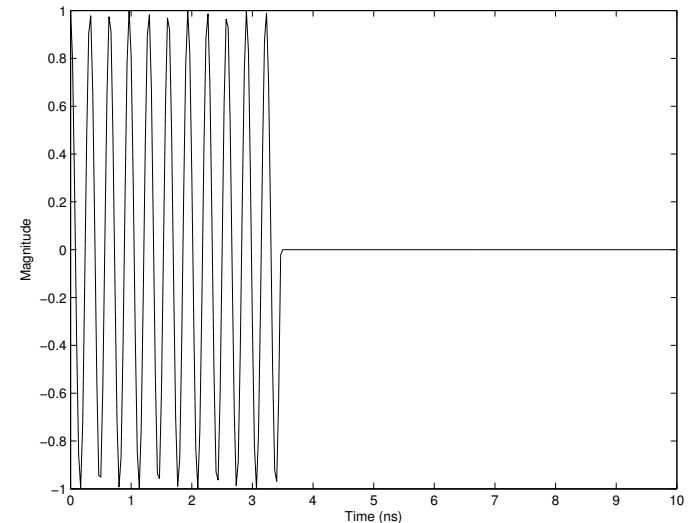

(a) single RF pulse

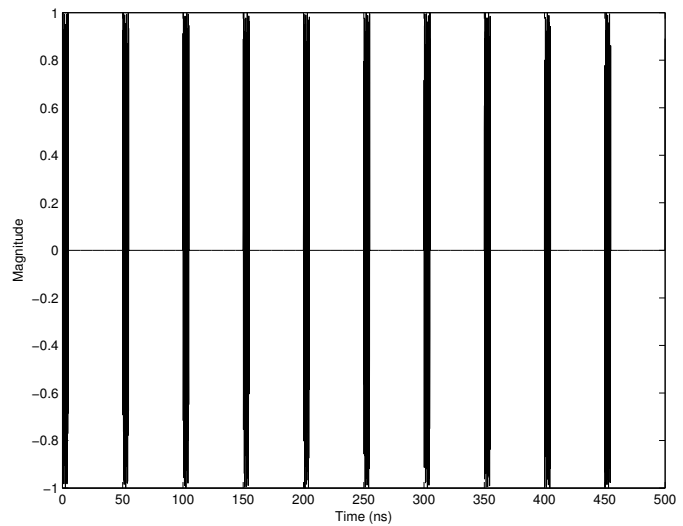

(b) pulse train

Figure 3.15: FMCW pulses 
The channel which the signal propagates through is simulated with a single block. The Transport Delay block internally generates a delay to simulate the physical delay as distance through air or coaxial cables. Other effects of the channel are ignored.

The receiver then receives $g(t)$, a time-shifted version of $f(t)$

$$
g(t)=f(t-\Delta t)
$$

The receiver also has a local source of $f(t)$. The two signals are mixed together as shown in the Product1 block, illustrated in Fig. 3.16(a). The effect of mixing two signals together is that the output contains harmonics of the sum and difference of the input frequencies. A filter can then be applied to remove the higher unwanted frequencies, as shown in Fig. 3.16(b). The resulting beat frequency is then,

$$
\Delta f=\Delta t \cdot k_{f}
$$

but for an actual system for measuring distance, the time passed corresponds with the distance the signal traveled,

$$
\Delta f=\frac{2 d}{c} k_{f}
$$

with a factor of two to account for the round trip path of the signal.

The signal's output frequency is used to calculate the equivalent distance using (3.8).

For the case where there is more than one return signal, then the output of the mixer will contain multiple beat frequencies. The beat frequencies will be visible on the output signal as the physical distance between the two objects are greater than the minimal resolution as given in (3.9). 


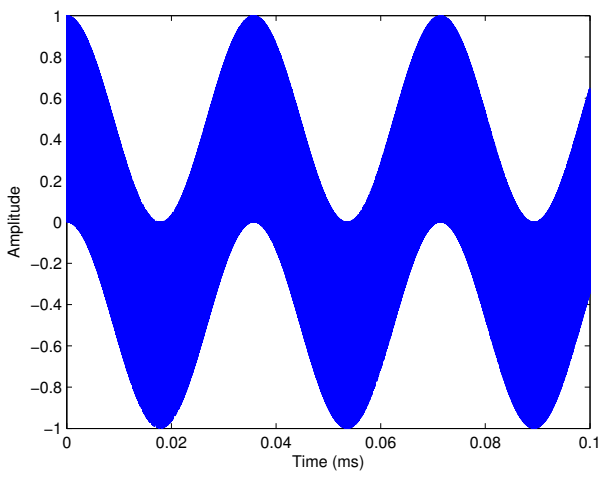

(a) mixer output

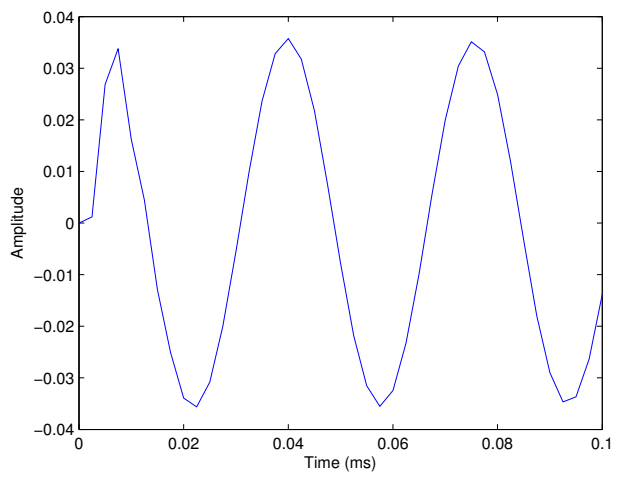

(b) filter output

Figure 3.16: FMCW output IF frequency 


\section{FMCW System}

A proposed FMCW system in Fig. 4.1 is a preliminary prototype to show the FMCW technique can be used to determine distance. This setup is used to take data to observe the length of coaxial cables, distance directly between two antennas, and distance with a reflection as shown in Fig. 4.2.

This system contains the minimal components needed to successfully operate the FMCW system. A function generator is used to externally trigger the network analyzer to begin sweeping and the oscilloscope to trigger and capture data. The fastest sweep rate of $6 \mathrm{~ms}$ is used with a sweep range of $3 \mathrm{GHz}$ to $4 \mathrm{GHz}$ or $1 \mathrm{GHz}$ of bandwidth. The application of a network analyzer is for $S$-parameter measurements. For this experiment, its internal YIG oscillator is used for a linear frequency ramp. The bi-directional coupler is used to split the signal from the network analyzer where one is directed to the antenna, and the other is to drive the LO port of the mixer. When the signal is returned it is amplified by one (or two) Avago LNA MMIC amplifiers and the oscilloscope is used to measure the signal. 


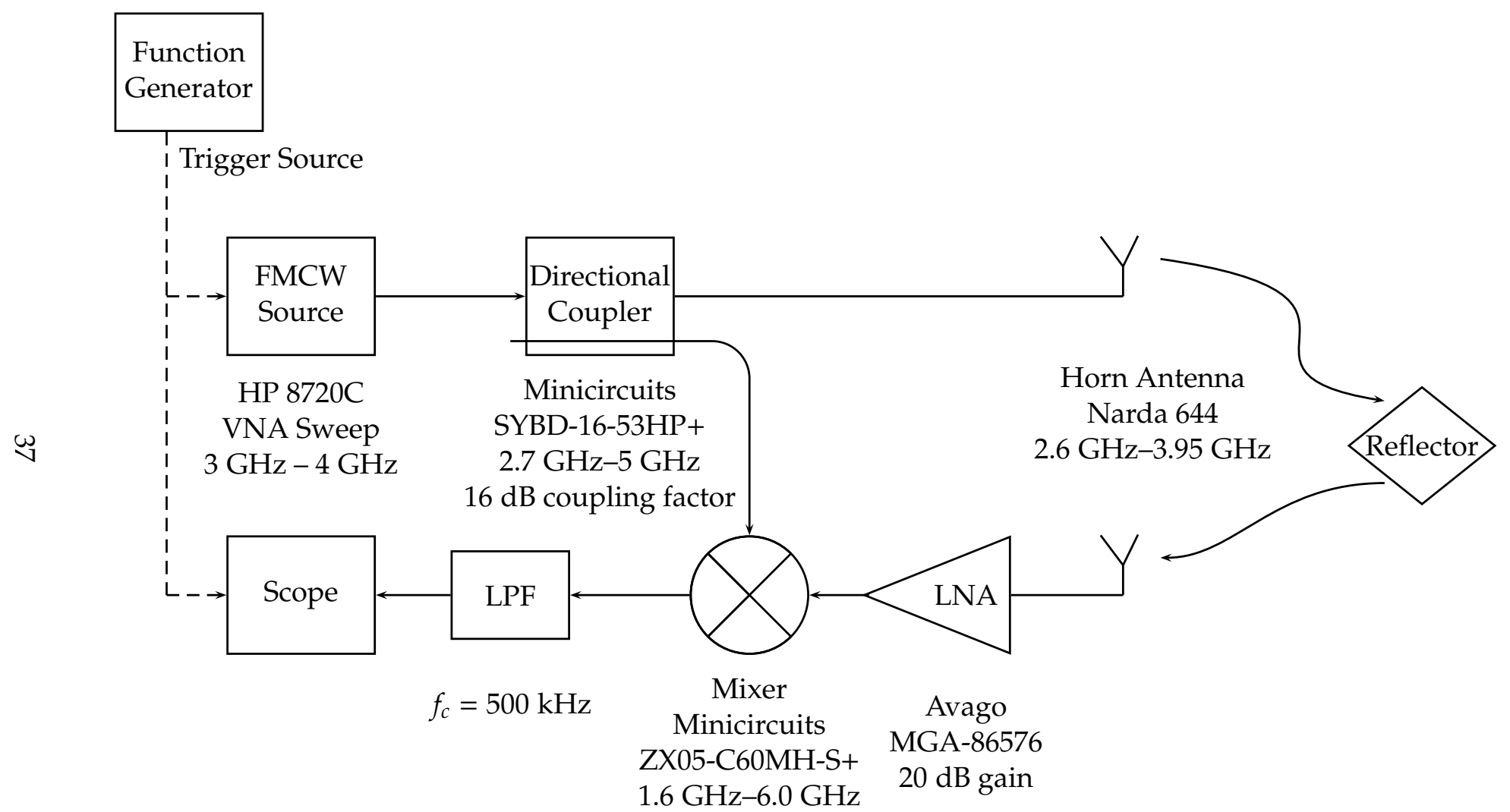

Figure 4.1: Block diagram for system test setup 


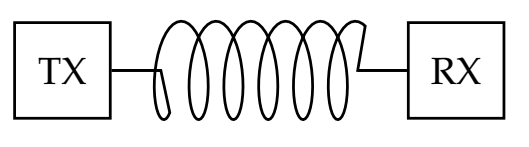

(a) RG-142 cables

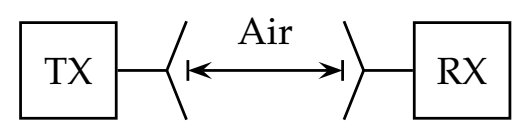

(b) Air

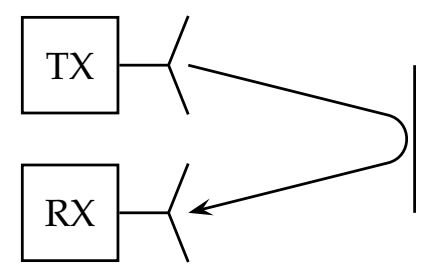

(c) Reflection

Figure 4.2: Various test setups used for characterizing the FMCW system

\subsection{Component Discussion}

Each component was characterized on the network analyzer to understand the function of the component along with its input and output characteristics.

\section{FMCW Source}

The HP 8719C network analyzer is used to provide the input signal source, it is capable of up to $20 \mathrm{GHz}$ output. [32] The network analyzer allows for a linear synthesized sweep ramp. By lowering the number of points measured to three, the total sweep time is minimized. The measurements by the network analyzer itself are not used. Thus, it does not have an impact on the distance measurements gathered. Also, by raising the IF bandwidth on the network analyzer, a sweep time of $6 \mathrm{~ms}$ can be achieved. 


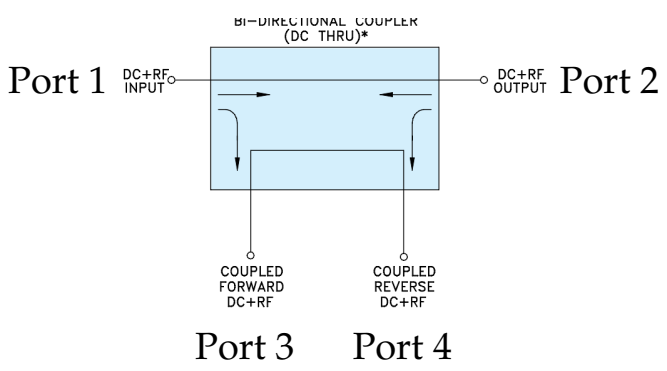

Figure 4.3: Schematic of bi-directional coupler [33]

\section{Bi-Directional Coupler}

The Minicircuits SYBD-16-53HP+ is a bi-directional coupler for $2.7 \mathrm{GHz}$ to $5.0 \mathrm{GHz}$ with a $16 \mathrm{~dB}$ coupling factor. Figure 4.3 shows the port configuration for the bidirectional coupler. The bi-directional coupler will pass signal through from port 1 to port 2 , and it will couple the port 1 signal to port 3 and port 2 signal to port 4 . The insertion loss is then

$$
\mathrm{IL}(\mathrm{dB})=10 \log \frac{P_{1}}{P_{2}}
$$

The coupling factor is the amount of signal that is transferred between the ports, thus

$$
\mathrm{CF}(\mathrm{dB})=10 \log \frac{P_{1}}{P_{3}}
$$

The S-parameters shown in Fig. 4.4 result from the operation of the bi-directional coupler. Unused ports are provided a $50 \Omega$ load during testing and operation for preventing unwanted reflections, specifically port 4 is unused in the system. The $S_{21}$ parameter shows insertion loss between $0.2 \mathrm{~dB}$ to $0.8 \mathrm{~dB}$. The $S_{31}$ parameter shows $17 \mathrm{~dB}$ coupling between port 1 and 3 , where the bi-directional coupler functions as a splitter. 


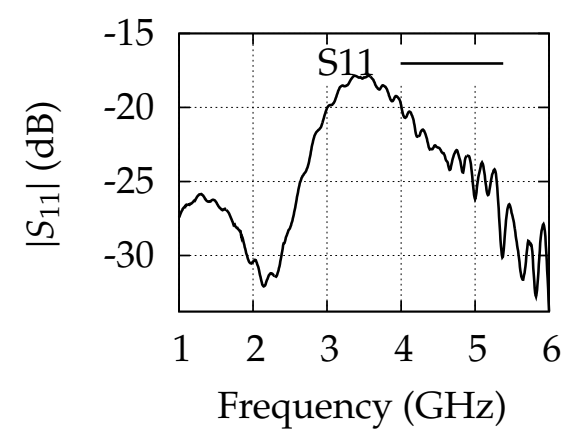

(a) $S_{11}$

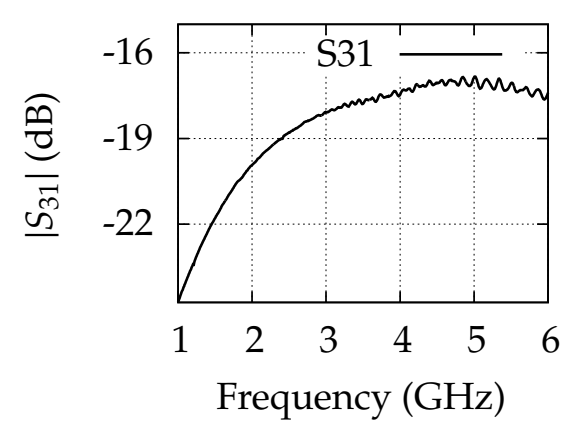

(c) $S_{31}$

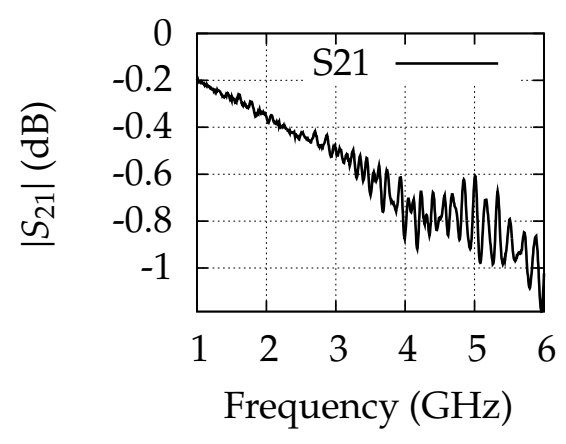

(b) $S_{21}$

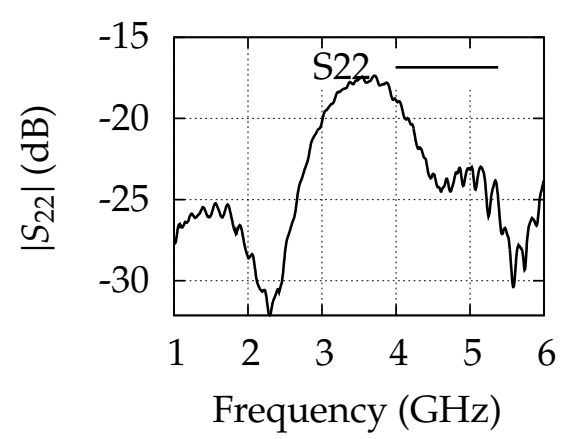

(d) $S_{22}$

Figure 4.4: S-parameters for the bi-directional coupler $2.7 \mathrm{GHz}$ to $5.0 \mathrm{GHz}$

\section{Mixer}

The mixer is a Minicircuits ZX05-C60MH+ for operation between $1.6 \mathrm{GHz}$ to $6.0 \mathrm{GHz}$ with a LO port power of $+13 \mathrm{dBm}$. The mixer was tested on a three-port network analyzer for $1.6 \mathrm{GHz}$ to $3 \mathrm{GHz}$. The S-parameters are shown in Fig. 4.5 and shows an insertion loss of the difference of power between the RF and IF ports, of $7 \mathrm{~dB}$. In Fig. 4.5(a), the IF port of the mixer is swept $10 \mathrm{MHz}$ below the RF frequency port. For Fig. 4.5(b), the LO port frequency is fixed at $1.6 \mathrm{GHz}$ for the duration of the sweep. The mixer can function with lower LO port power at the expense of increased insertion loss. 


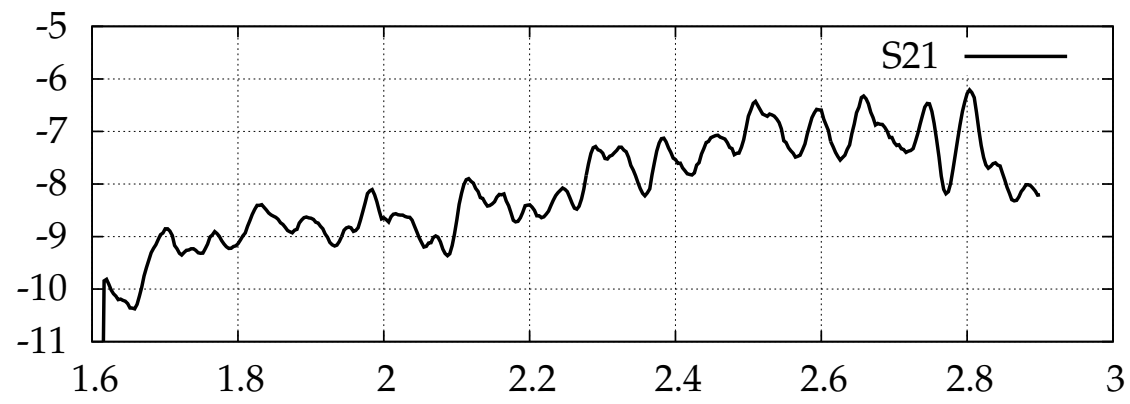

(a) Fixed IF at $f_{\mathrm{RF}}-10 \mathrm{MHz}$

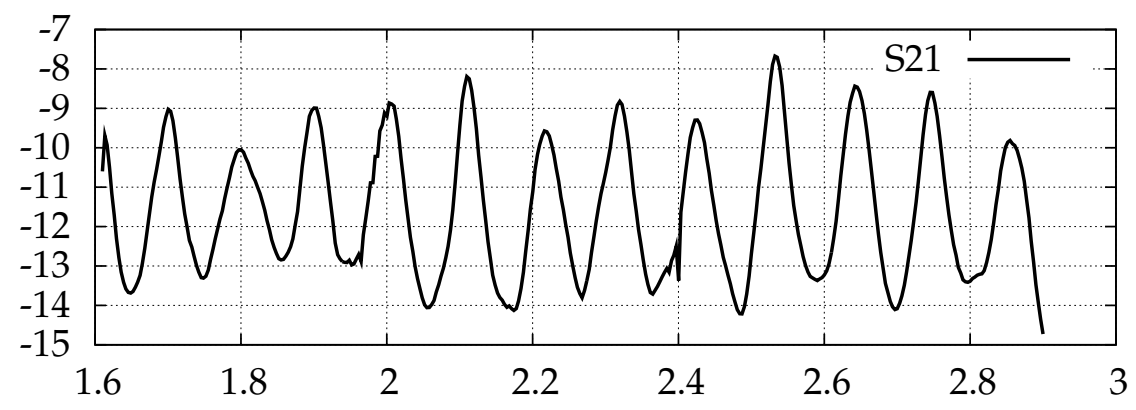

(b) Fixed $\mathrm{LO}$ at $1.6 \mathrm{GHz}$

Figure 4.5: Mixer insertion loss with LO port power of $+10 \mathrm{dBm}$

\section{Low-Noise Amplifier}

The low-noise amplifier (LNA) used is Avago MGA-8657, a monolithic microwave integrated circuit (MMIC). The $S$-parameters in Fig. 4.6 shows a $\left|S_{21}\right|$ gain of $20 \mathrm{~dB}$ for ID:2, while the gain for ID:1 is $10 \mathrm{~dB}$. The LNA IC was hand soldered to a demo board provided by Avago. The datasheet states that the amplifier has $23 \mathrm{~dB}$ gain at $4 \mathrm{GHz}$ but it is also rated for applications between $1.5 \mathrm{GHz}$ to $8 \mathrm{GHz}$. The LNA is designed to be used without an input or output matching network for a $50 \Omega$ system, although the datasheet provides for matching for low noise figure. The difference in gain may be attributed to soldering work, damage to the part, or manufacturing tolerances. 

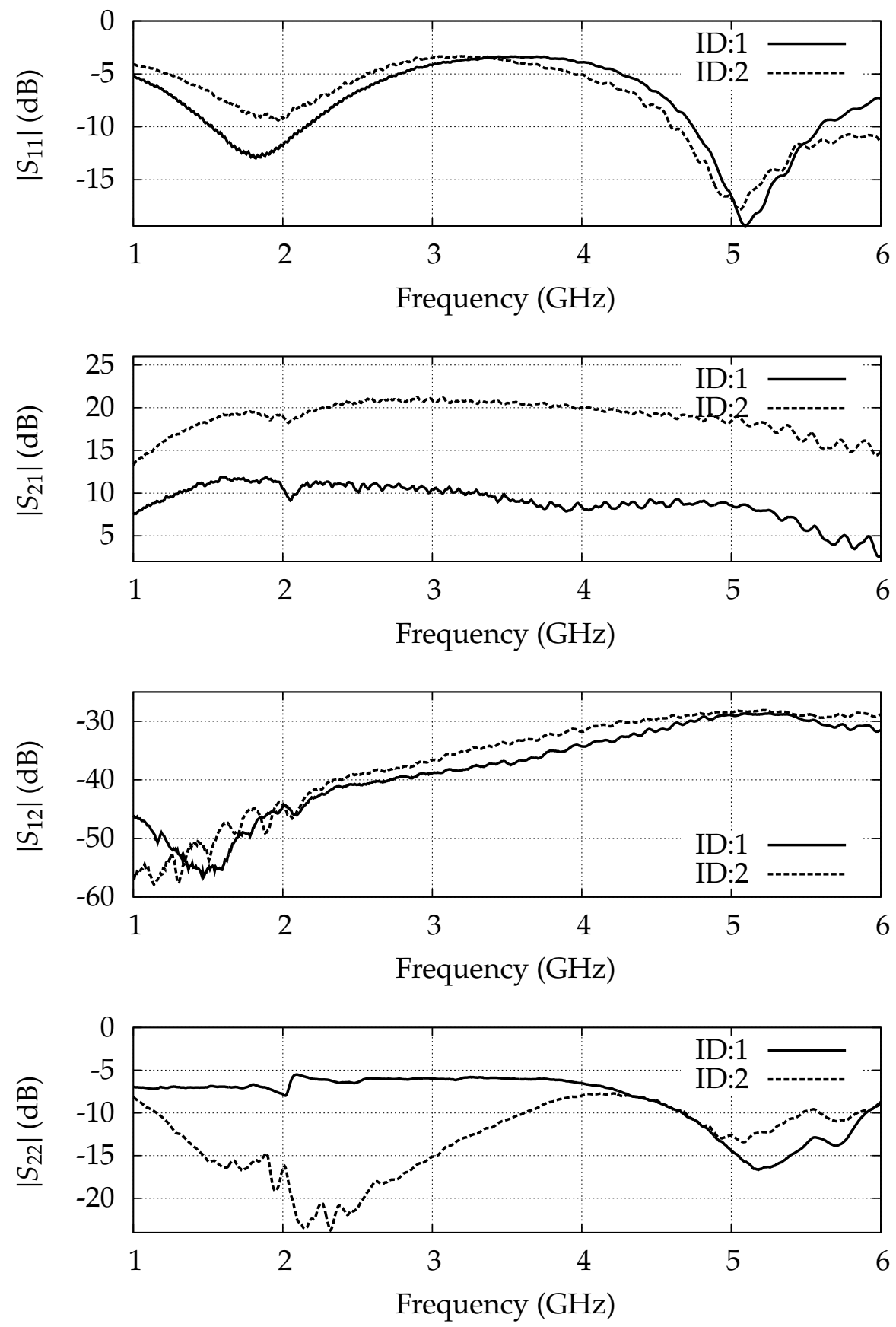

Figure 4.6: S-parameters for LNA 


\section{Horn Antenna}

The horn antenna is the Nada 644 covering $2.60 \mathrm{GHz}$ to $3.95 \mathrm{GHz}$. In this band range it's max VSWR is 1.15. The datasheet [34] shows a gain of at least $15 \mathrm{dBi}$ with respect to an ideal isotropic antenna, for frequencies higher than $2.65 \mathrm{GHz}$. A horn antenna is a directive antenna which can only detect signals incoming to the front opening as it functions as a waveguide. The physical dimensions of the antenna are approximately 16 in. by $10 \mathrm{in.} \mathrm{by} 7 \mathrm{in}$.

\subsection{Test Setup}

A photo of the reflection test setup is in Fig. 4.7. Visible is the VNA which acts as the FMCW source, which is connected to the bi-directional coupler to split the signal to the transmit antenna and a local copy sent to the mixer. Horn antennas are placed facing the reflector for the receive antenna to sense the reflected signal from the transmit antenna. The signal is received and amplified by LNAs (not visible), powered by the DC power supply. The resulting IF output of the mixer is then viewed on the oscilloscope.

In Fig. 4.8, the back view of the setup is shown. The LNAs are present on the test bench, and also the trigger source. The trigger source is a low frequency square wave output used for controlling the oscilloscope and network analyzer. The trigger source starts the sweep on the network analyzer, and it also triggers the oscilloscope to begin capturing data.

A closer view of the test board containing the mixer and bi-directional coupler is shown in Fig. 4.9. The components are secured on a board to maintain consistent output results. Port 4 of the bi-directional coupler for reverse coupling with port 2 contains a matched $50 \Omega$-load. The physical dimensions of the bi-directional coupler 


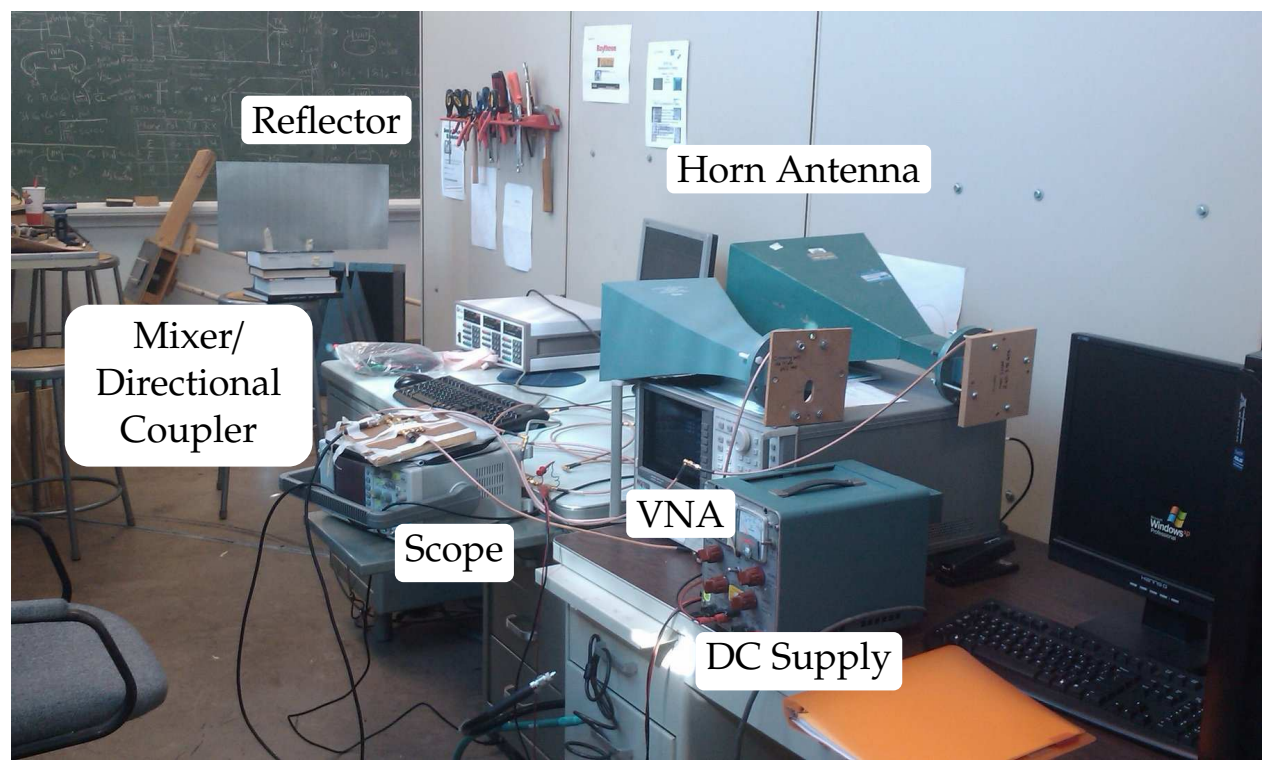

Figure 4.7: Front view of test setup for distance measurements with a reflector

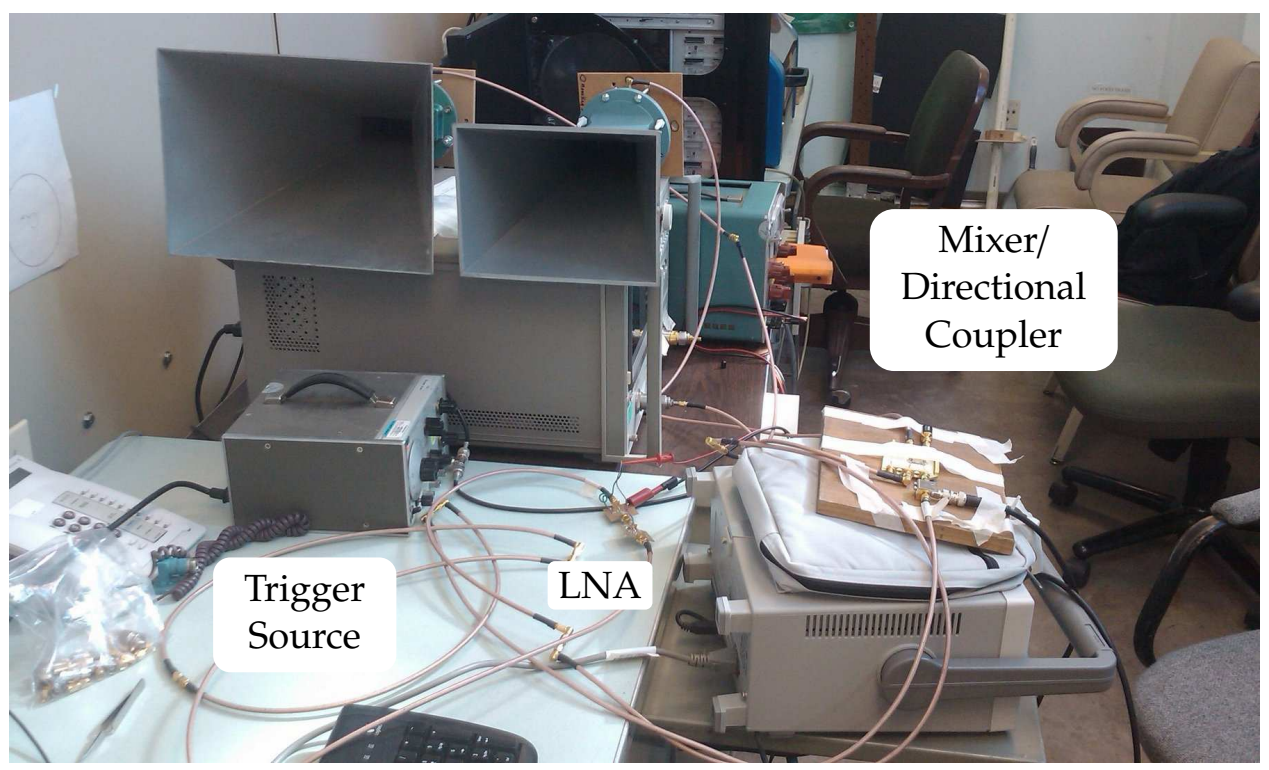

Figure 4.8: Back view of test setup for distance measurements with a reflector 


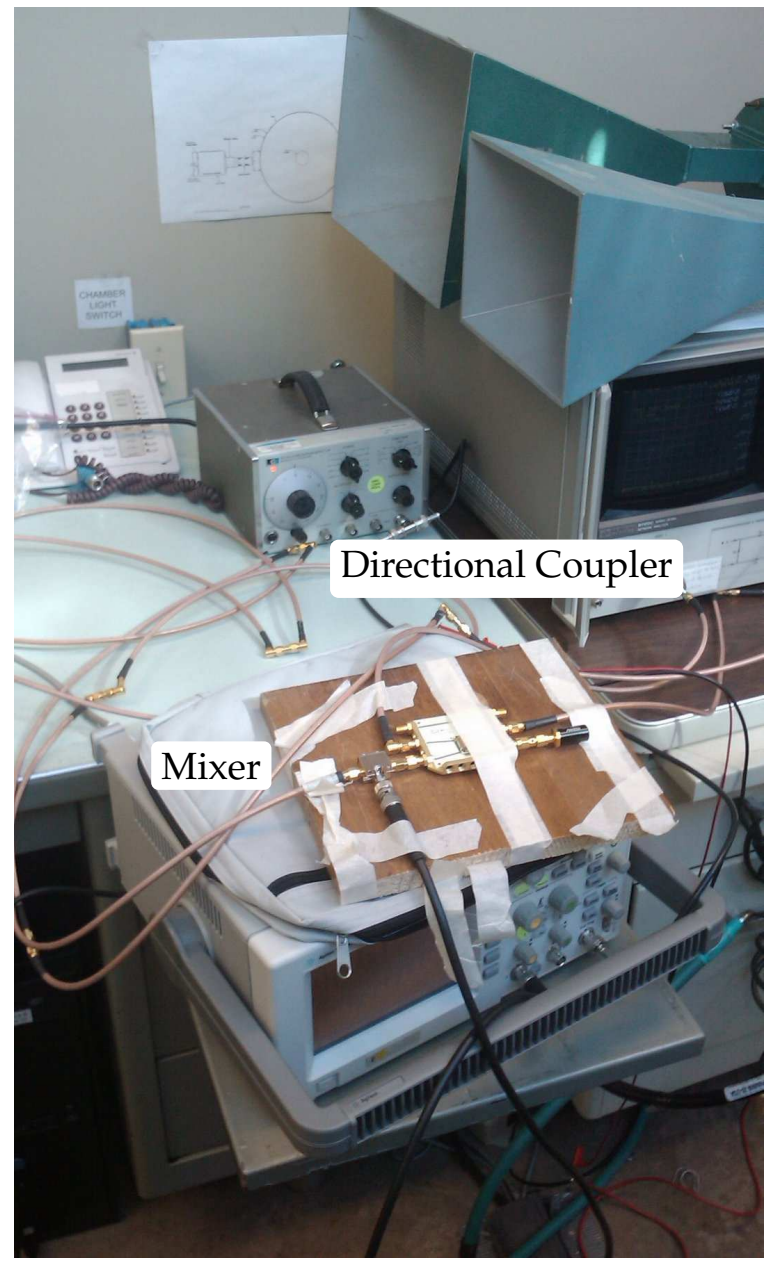

Figure 4.9: Test board containing the mixer and bi-directional coupler

and mixer allows them to be directly connected together via a through-line.

In Fig. 4.10, two LNA's boards are chained together to allow for adequate gain such that the oscilloscope can detect the beat signal at the IF port of the mixer. The two amplifiers are powered by the same 5 V DC supply. 


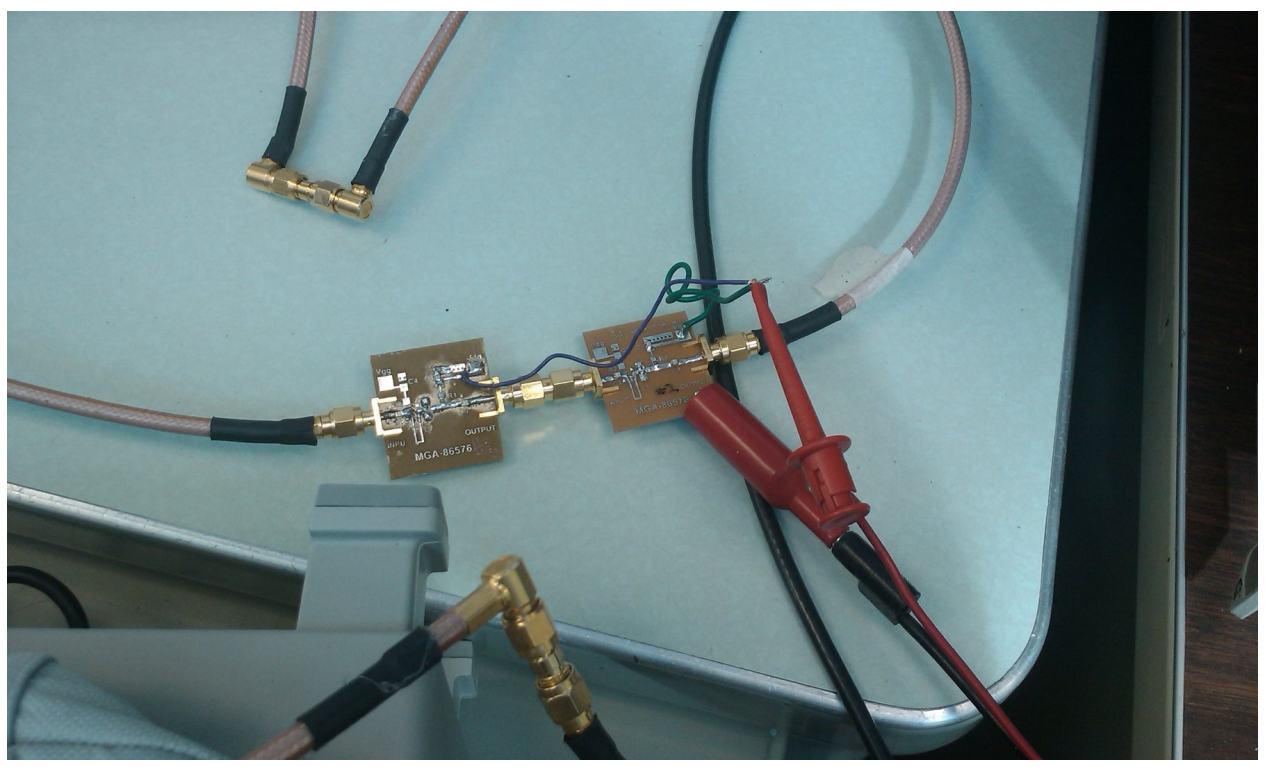

Figure 4.10: Two stage amplifier connected between the receive antenna and mixer

\subsection{Data Discussion}

In this section the results from the experimental measurements are shown. Measurements were taken with an Agilent DSO3000 series oscilloscope. Data capture via USB was used to record data for plotting purposes, and to integrate with MATLAB for analyzing the signal in the frequency domain. Measurements for three test cases shown earlier in Fig. 4.2, were considered and analyzed.

\section{Oscilloscope Output}

The output waveform from the mixer is shown in Fig. 4.11 for a cable length measurement, Fig. 4.12 for a distance measurement in air, and Fig. 4.13 for a reflection measurement. The beat frequency of the three figures increase due to difference in lengths measured. With longer distances, the beat frequency increases. The peak to peak voltage of the signal decreases not only due to increasing distances, but from the effects due to the channel. Figure 4.11 has the highest amplitude since the signal 


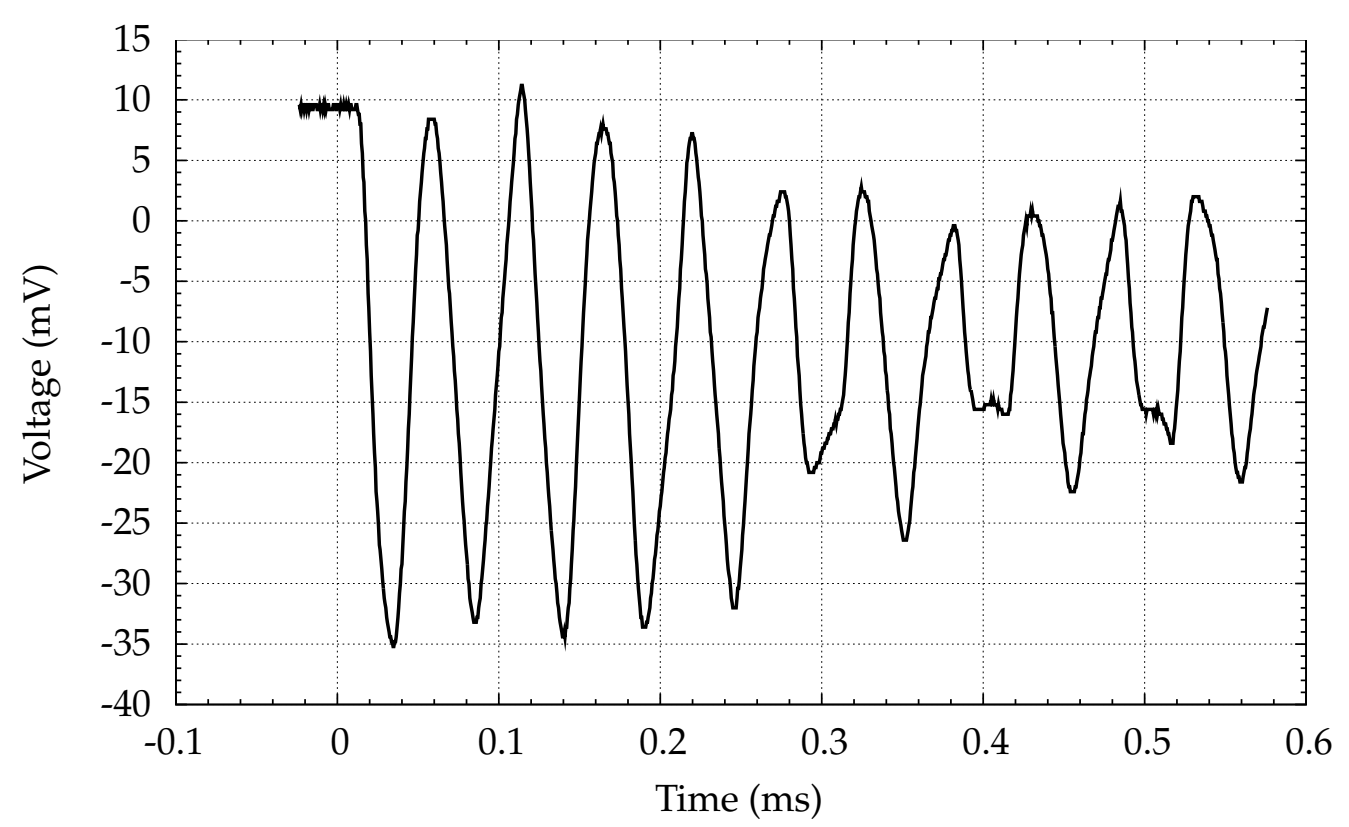

Figure 4.11: Oscilloscope output for a coaxial cable length of $120 \mathrm{~cm}$

was contained in coaxial cables. Figure 4.12 has a higher signal power compared with Fig. 4.13 as the latter has extra power loss from an reflection. In Fig. 4.11, the amplitude of the signal varies over time. At the start of the sweep the network analyzer outputs a $3 \mathrm{GHz}$ signal, and at the end of the sweep a $4 \mathrm{GHz}$ signal is outputted, since the system components have varying frequency responses, when the network analyzer is outputting higher frequencies, the amplitude is attenuated.

In Fig. 4.14 the fast Fourier transform (FFT) of five measurements, including the measurement from Fig. 4.11 is shown. From the spectrum, the plot shows a large spike at $4 \mathrm{kHz}$. The frequency measurement was made by finding the peak value, ignoring any dc offset, and then creating a weighted average value using the peak and the value left and right of the peak. This weighted average is calculated five times, and then the results are averaged together. The resulting FFT spectrum for each trial was consistent such that each measurement overlaps with each other. Since the propagation channel was within coaxial cables the measurement was stable. 


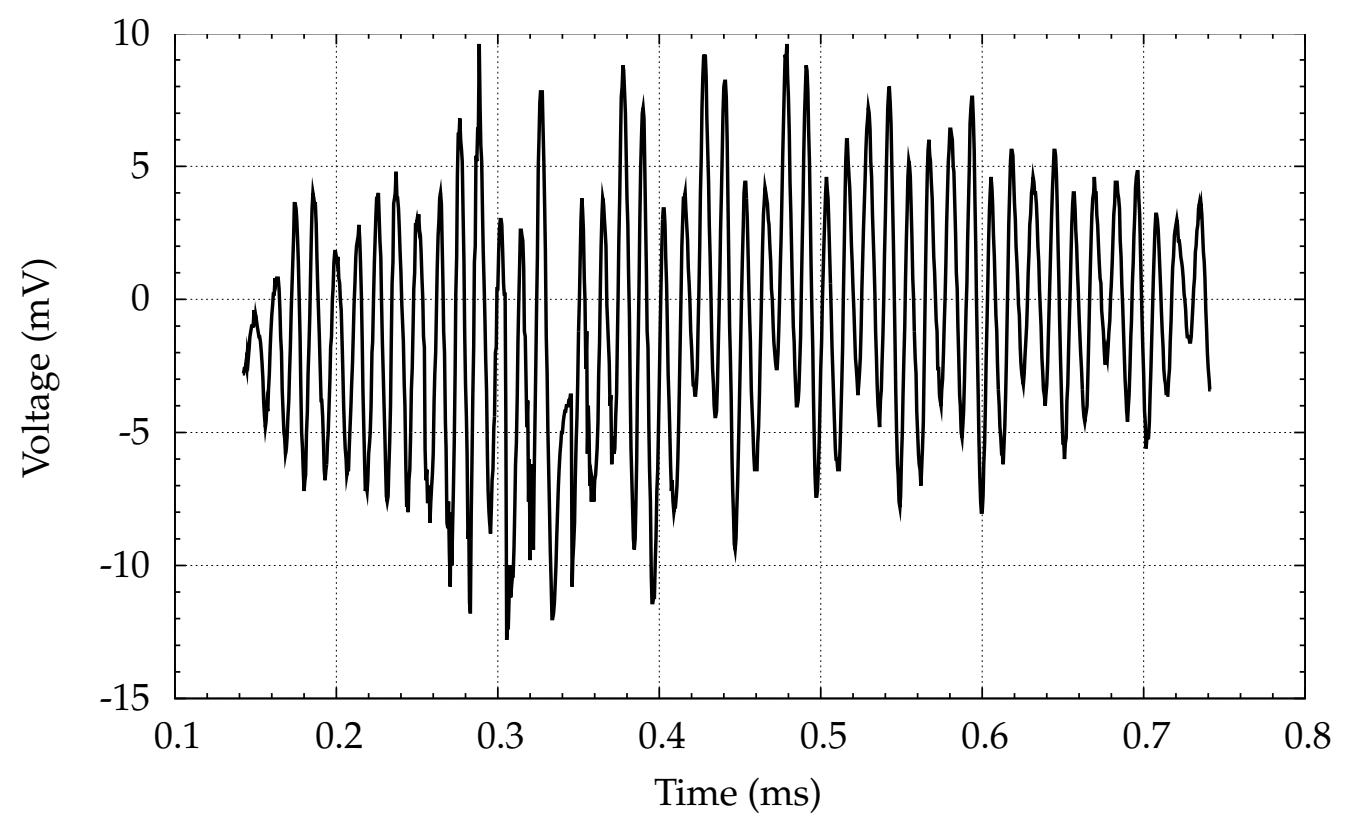

Figure 4.12: Oscilloscope output for a distance $272 \mathrm{~cm}$ through air

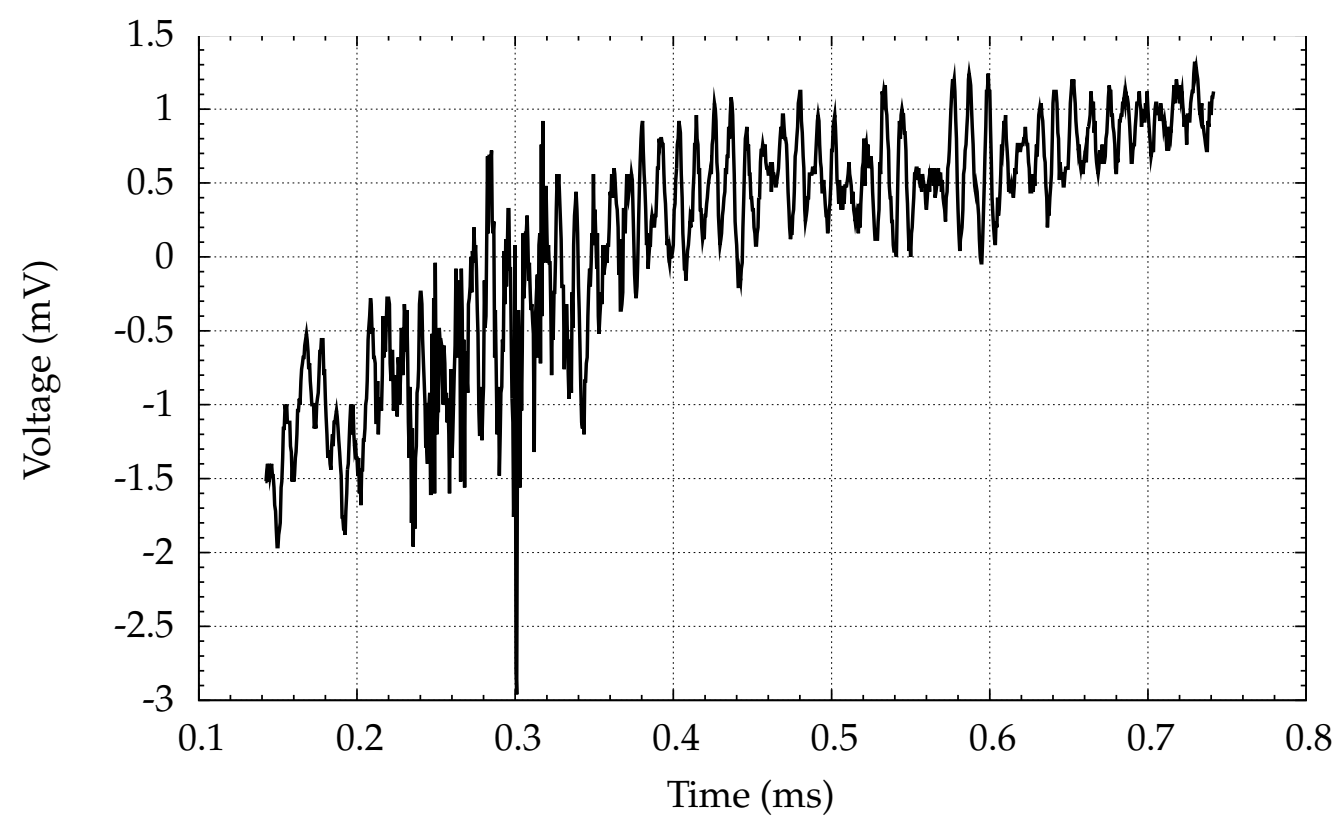

Figure 4.13: Oscilloscope output for a distance $528 \mathrm{~cm}$ in air with a reflection 


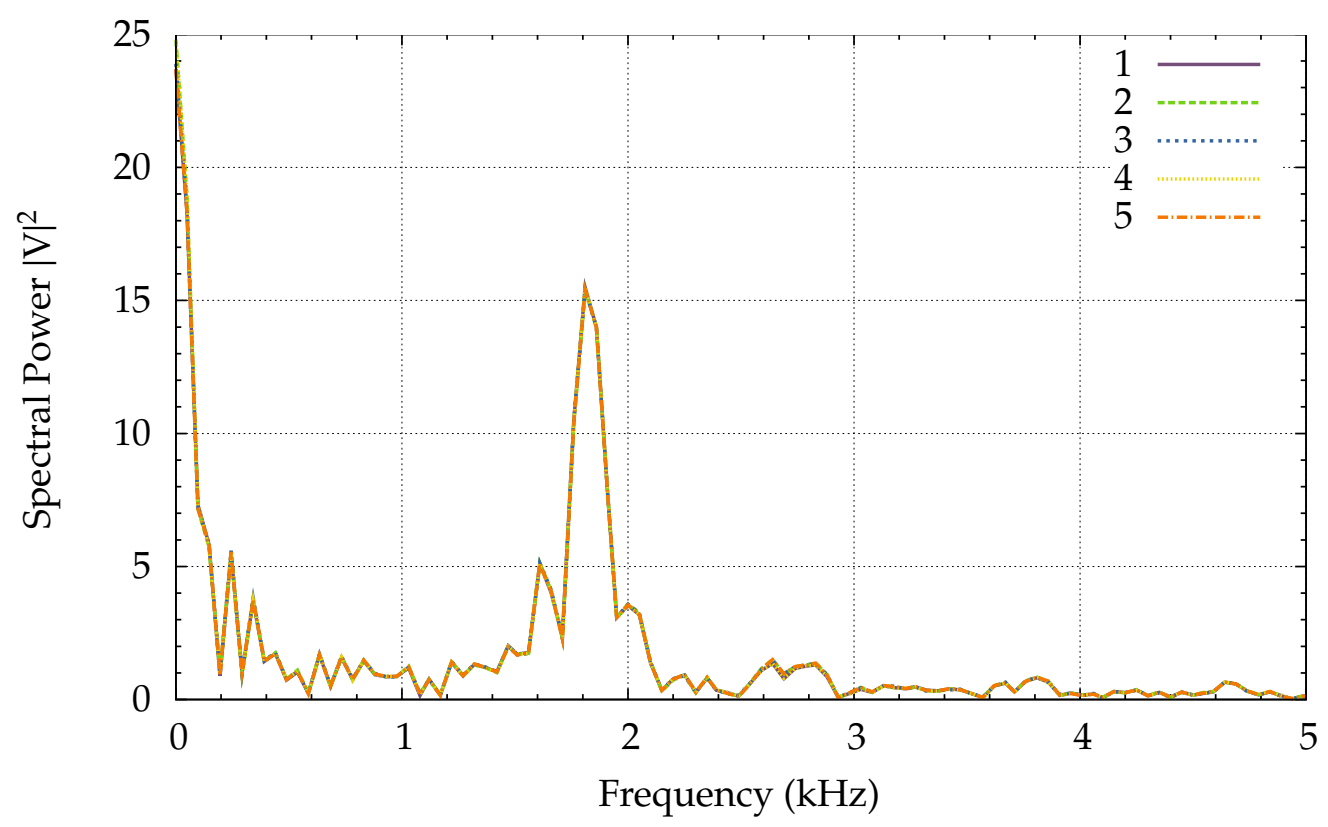

Figure 4.14: Spectrum of five samples for a cable length of $120 \mathrm{~cm}$

Similarly, in Fig. 4.15, the spectrum shows the FFT result for five measurements for a distance of $528 \mathrm{~cm}$ including a reflection. Unlike the measurement through coaxial cables, variations occurred when' the signal propagated through air. Other relative maximums show reflections from the test environment.

\section{Length Measurements}

The results from the three test cases of measuring coaxial cable length, distance through air, and round trip distance including a reflection will be shown. The different slopes in the regression models of different cases are discussed, and the different system offset values are also analyzed.

\section{Cable Length}

The first experiment that was done was to characterize various length of RG-142 cable. RG-142 have a typical velocity factor of $70.9 \%$ [35], this gives a propagation 


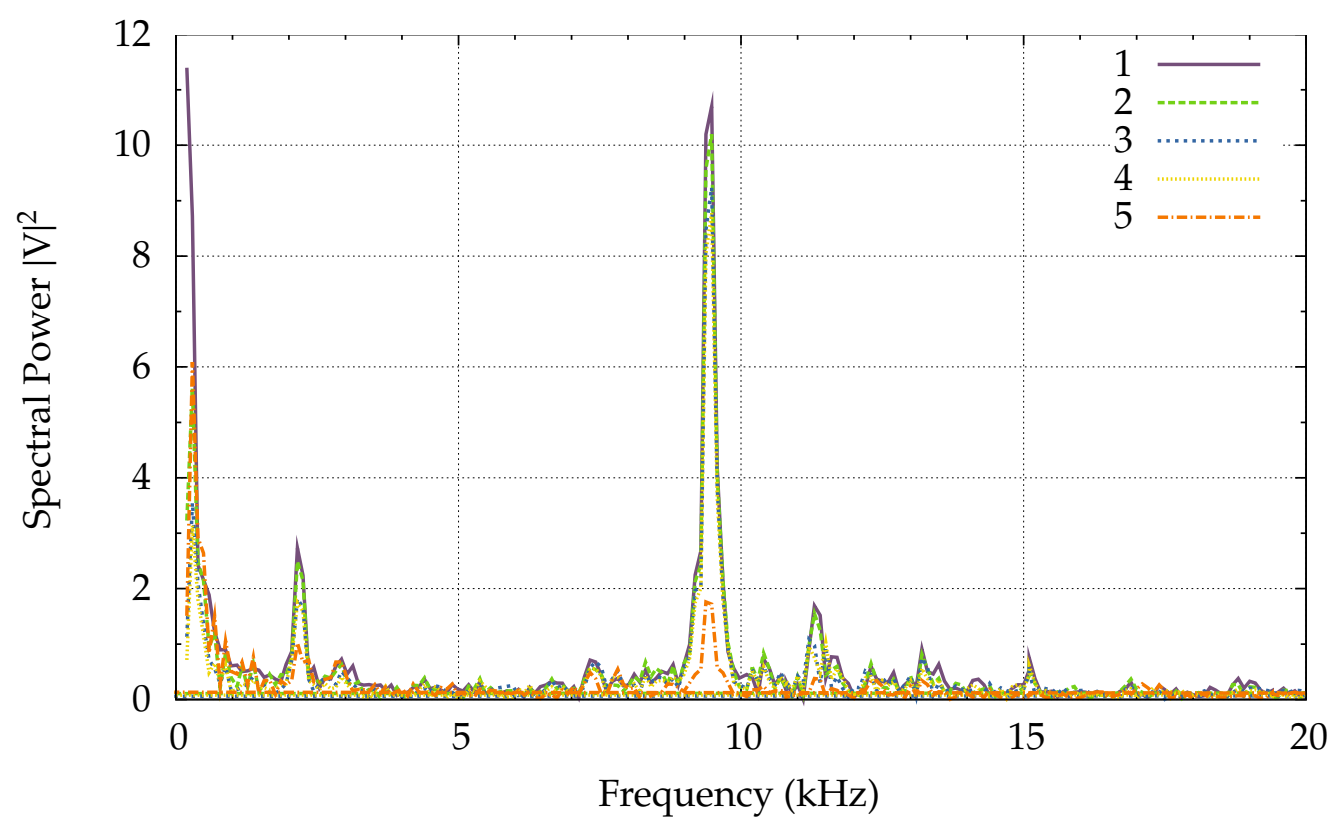

Figure 4.15: Spectrum of five samples of a distance $528 \mathrm{~cm}$ in air with a reflection

factor of $2.126 \times 10^{8} \mathrm{~m} / \mathrm{s}$. Using the network analyzer, each $60 \mathrm{~cm}$ cable has a loss of $1 \mathrm{~dB}$. The data was collected by adding cables in-between the transmitter and receiver. The results in Fig. 4.16 show a consistent slope for an increasing cable length. The output frequency is

$$
\Delta f=k_{f} t=k_{f}\left(d / v_{p}\right)
$$

with $d=1 \mathrm{~m}$ then $\Delta f$ is the theoretical slope for the experiment, where $k_{f}$ is the sweep source constant as defined in (3.5.

The second test added an amplifier in the receiver chain; the net effect is an increased offset distance from the extra length. The amplifier is fed via $50 \Omega$ lines and also contains a microstrip line that acts as an inductor for matching. Figure 4.17 shows the PCB layout and schematic of the LNA with the matching network. The physical length of the board and inductor introduces delay into the system. The thin "U" shape microstrip is bridged into the circuit with solder. The total effect is an 


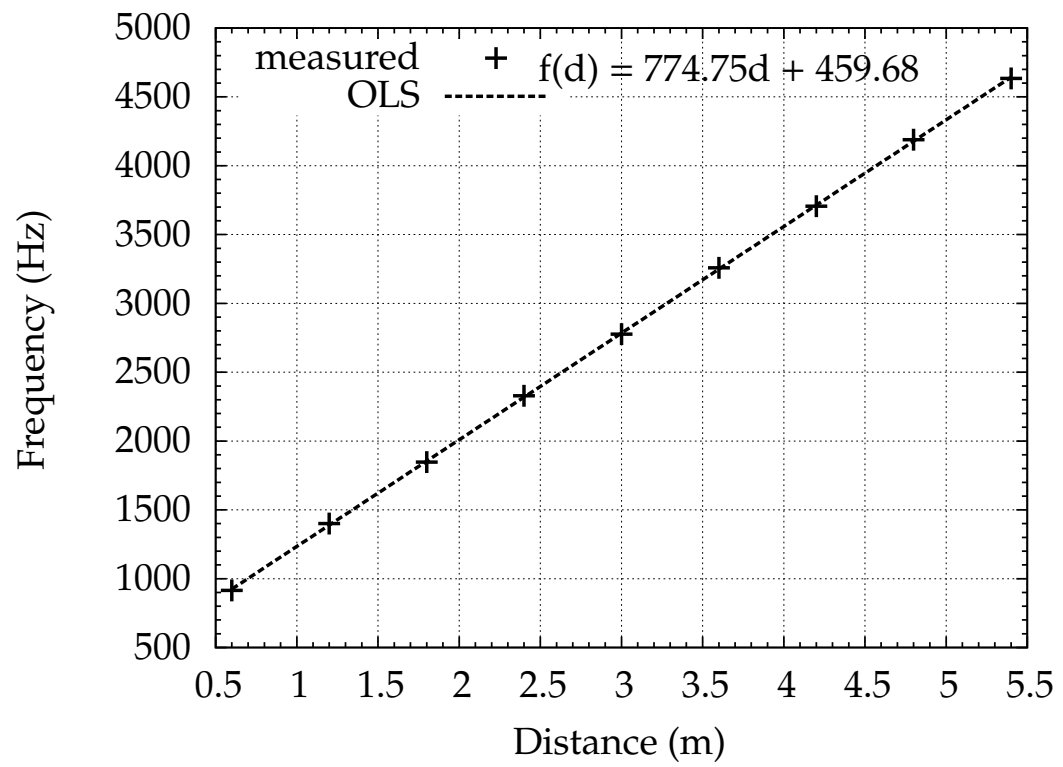

Figure 4.16: Output frequency using different cable lengths

increase of a few centimeters in the system path. In Fig. 4.18 , the least-squares fit shows that the slope is similar, but there is a $70 \mathrm{~Hz}$ difference in the $y$-intercept point. The slope is similar as it is defined by the $k_{f}$ value which is related to the sweep bandwidth and sweep time, along with the propagation velocity of the channel. These values remained constant for the two measurements.

\section{Distance Measurements}

The third and fourth tests used the exact same system. In Fig. 4.19, the distance was directly measured between the two horn antennas, while in Fig. 4.20 the total round trip distance was measured to a metallic sheet acting as a reflector. From the experiment, there is no noticeable difference in the results between the two systems. The $y$-intercept in the least-square regression remains the same as the system did not change. The slopes in the two systems are also the same. The slope differs from the previous cable measurements as the propagation velocity in the cables is $0.709 c$, 


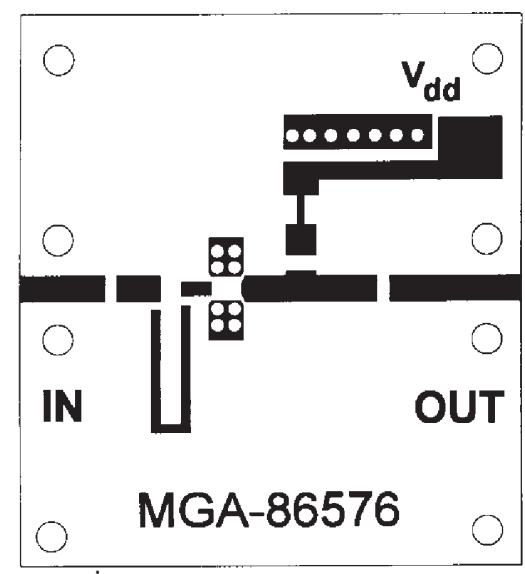

(a) PCB layout

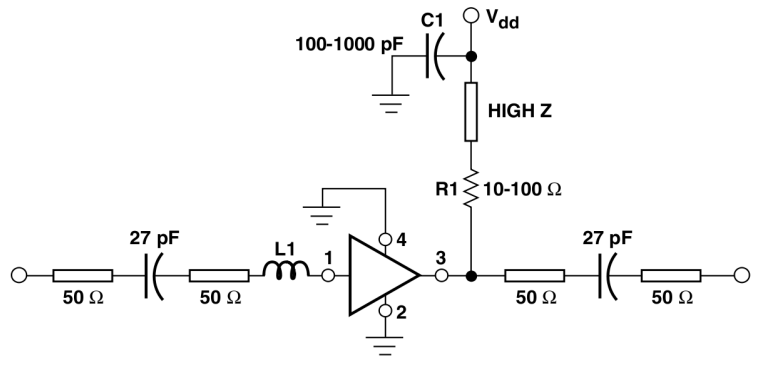

(b) Schematic

Figure 4.17: LNA circuit schematic and layout from datasheet [36]

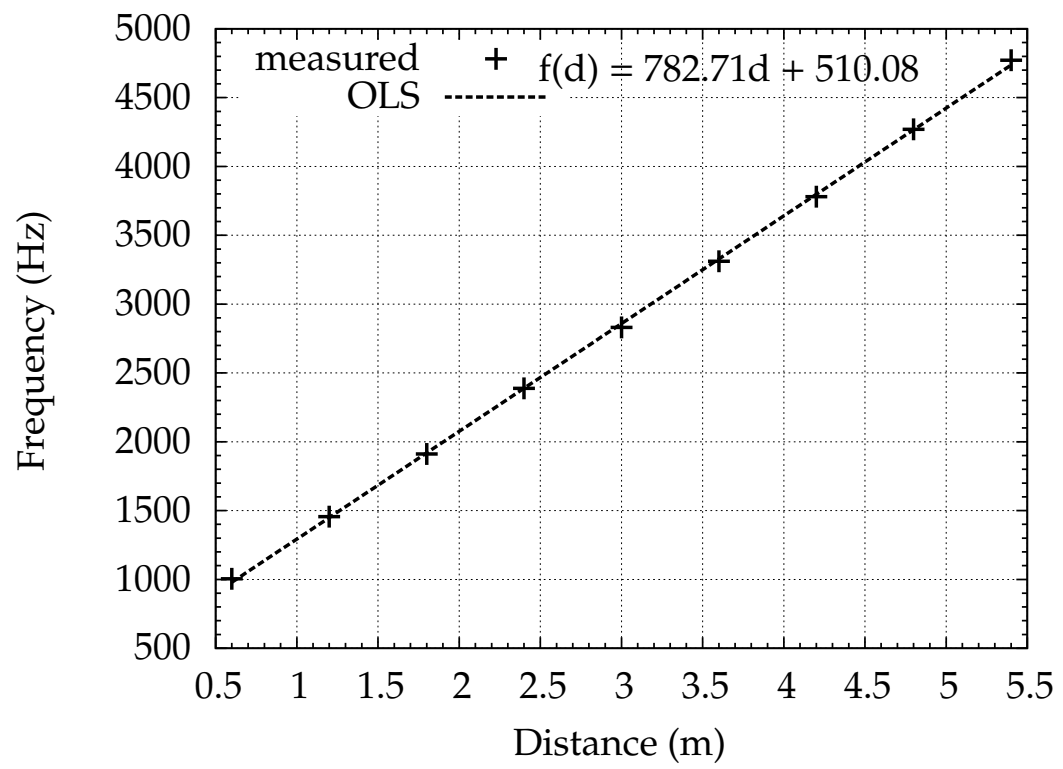

Figure 4.18: Output frequency with using different cables length and an amplifier 


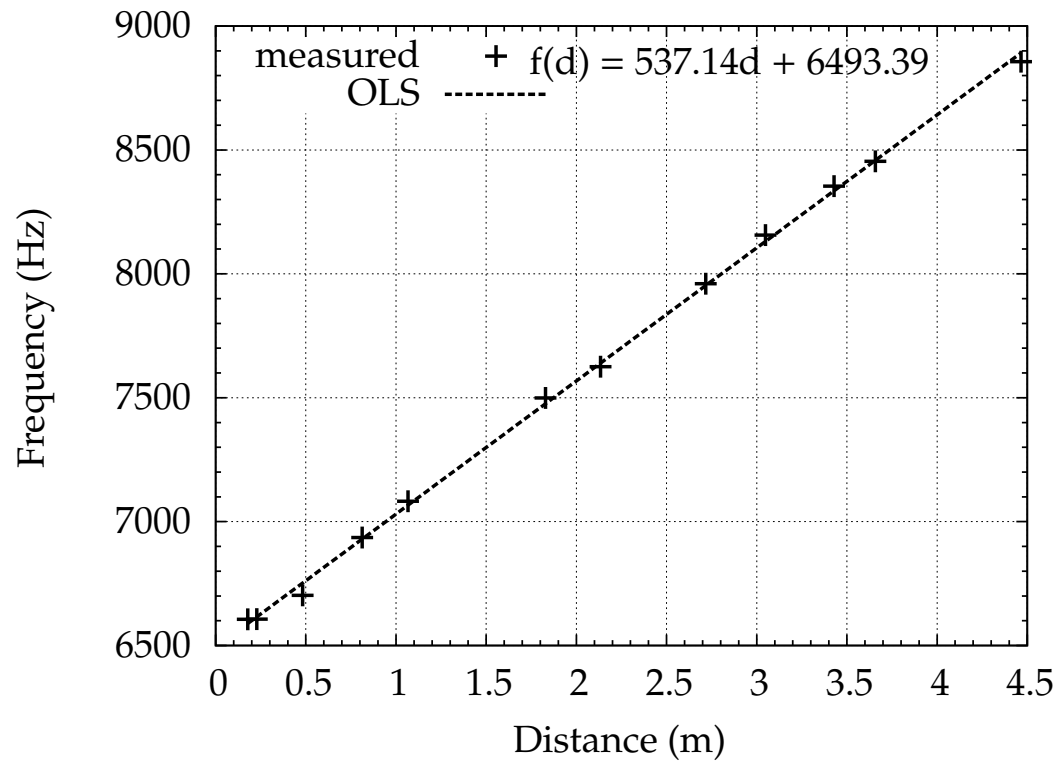

Figure 4.19: Output frequency from signal transmitted through air

compared with propagation via air which is $c$.

\subsection{Ordinary Least Square Model}

The theoretical slope from the ordinary least-squares (OLS) regression can be found by

$$
m=\frac{k_{f}}{v_{p}}
$$

where from (3.5)

$$
\begin{aligned}
k_{f} & =\frac{B W}{T_{\text {sweep }}}=\frac{1 \mathrm{GHz}}{6 \mathrm{~ms}} \\
& =1.667 \times 10^{11} \mathrm{~Hz} / \mathrm{s} .
\end{aligned}
$$




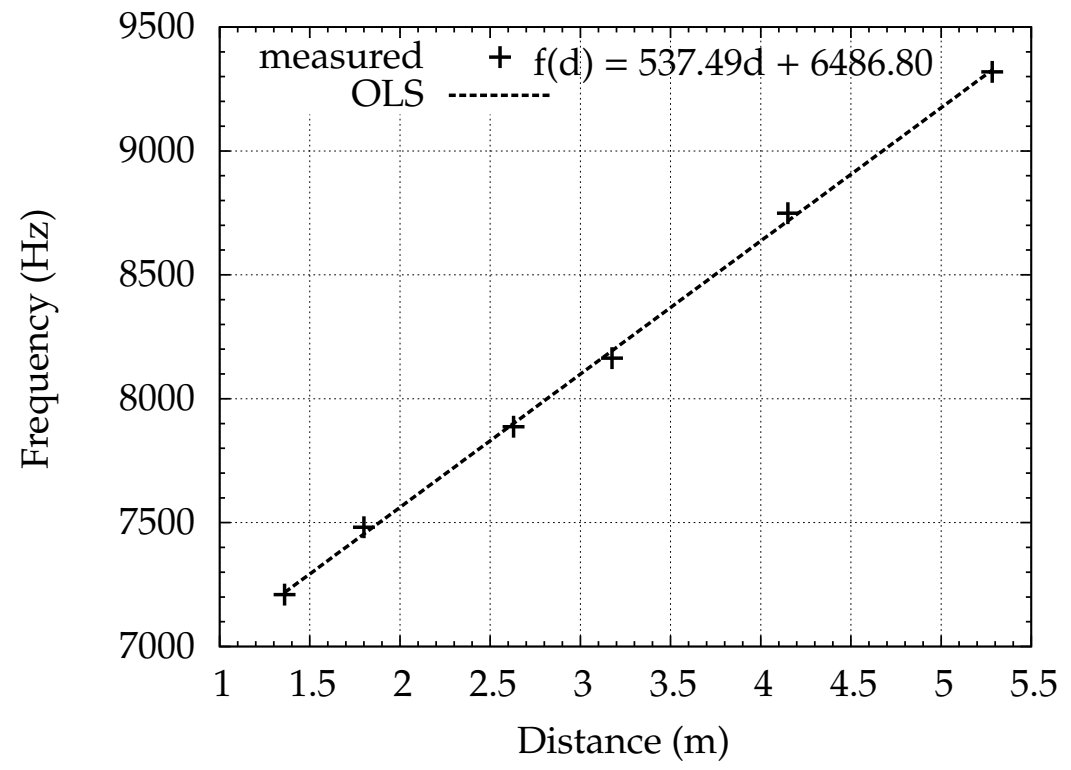

Figure 4.20: Output frequency from signal transmitted through air with a reflection

Thus, the corresponding slope values for RG-142 is

$$
\begin{aligned}
m_{\mathrm{rg}} & =\frac{k_{f}}{v_{p}}=\frac{1.667 \times 10^{11} \mathrm{~Hz} / \mathrm{s}}{2.099 \times 10^{8} \mathrm{~m} / \mathrm{s}} \\
& =794.2 \mathrm{~Hz} / \mathrm{m}
\end{aligned}
$$

and for air is

$$
\begin{aligned}
m_{\mathrm{c}} & =\frac{k_{f}}{c}=\frac{1.667 \times 10^{11} \mathrm{~Hz} / \mathrm{s}}{2.998 \times 10^{8} \mathrm{~m} / \mathrm{s}} \\
& =556.0 \mathrm{~Hz} / \mathrm{m} .
\end{aligned}
$$

The experimental value for RG-142 from Fig. 4.18 is 782.71 , and for air is 537.14 and 537.49 from Figures 4.19 and 4.20 . In both cases, the experimental value is lower than the expected theoretical slope. A difference of $80 \mathrm{~Hz}$ in RG-142 and $55 \mathrm{~Hz}$ in air is an error of approximately $10 \mathrm{~cm}$.

The theoretical slope value is determined from $k_{f}$, which in turn is from the FMCW source bandwidth and sweep time. The bandwidth and sweep time of the 
FMCW should be precisely measured to confirm if the error arose from it or other system components.

The FMCW system can be extended to longer distances as long as the receiver is able to detect the signal. FMCW is a linear sweep, thus any increased distance will lead to an increase of frequency difference. The fundamental limit of distance in the system is based upon the sweep time of the chirp signal. There must be enough time for the transmitted signal to be returned, before the chirp signal begins a new sweep, otherwise the FMCW source frequency becomes invalid.

With the FMCW system, a distance can be measured. This distance measurement is important for boundary detection. As shown in the OLS models, there is an offset value arising due to the signal path between the different components and to the antenna. The offset value is the zero distance measurement.

\subsection{Identification}

A real environment contains multiple sources of RF reflectors. For the receiver to uniquely identify the location of a node, a fingerprint type spectrum output is needed. A unique reflector is used to allow for this purpose. A simple solution is to have two reflectors spaced out at a known distance, then the resulting response can search for two peaks spaced apart with the corresponding distance. A possible real environment scenario is illustrated in Fig. 4.21. Reflections from environmental clutter or walls can then be ignored to get the correct distance measurement. Other environment objects generally will not have the exact spacing of the unique reflector.

Figure 4.22 shows the experiment performed with the special reflector spaced apart by $25.4 \mathrm{~cm}$. The spectrum clearly shows two peaks The peak at zero shows a dcoffset in the received signal. The other peaks show the reflection from the background environment or reflections from within the circuit itself due to impedance mismatches. 

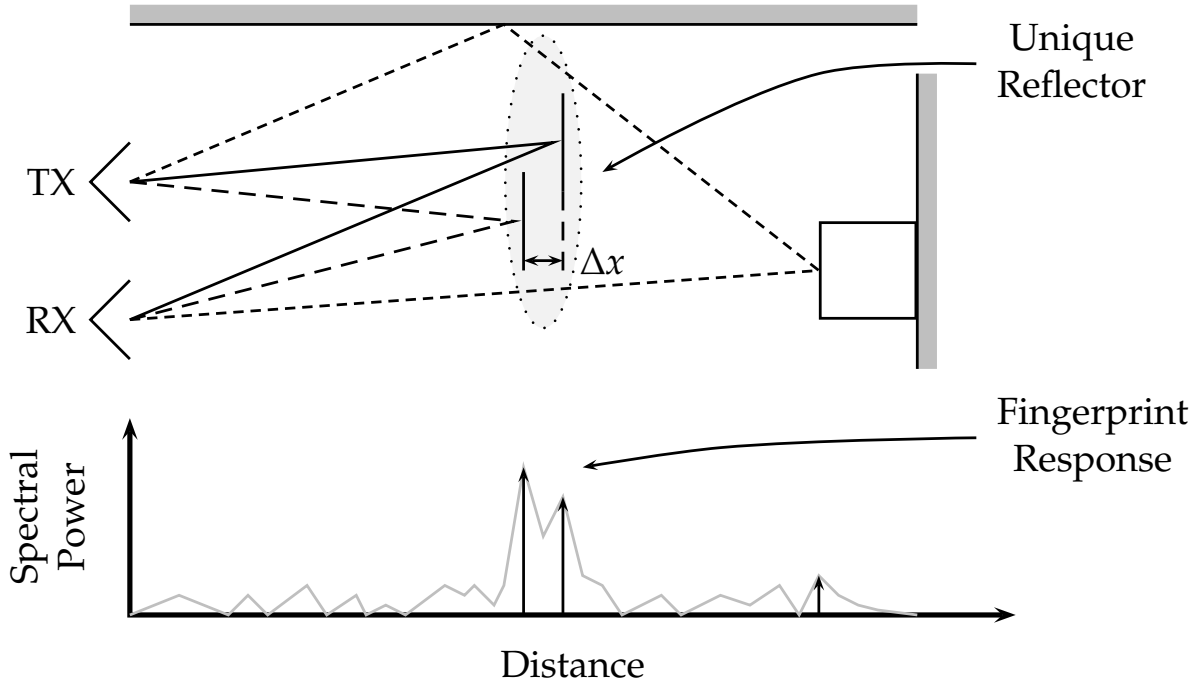

*Not to scale

Figure 4.21: A plausible response from a FMCW system with a unique reflector within a real environment.

The seperatation of the two peaks is $25.9 \mathrm{~cm}$ or a difference of $0.5 \mathrm{~cm}$. This value is close to the measured value. The two plates of the reflector were not perfectly parallel, thus the variation in this tolerence and the accuracy of the measurement itself could account for the error.

A uniquely spaced reflector is an adequate method for unique identification. The identification problem is now shifted to having two objects in the background which are spaced at a distance equal to the unique reflector. A possible solution is to also consider the relative received power level from the reflector to further narrow errors. 


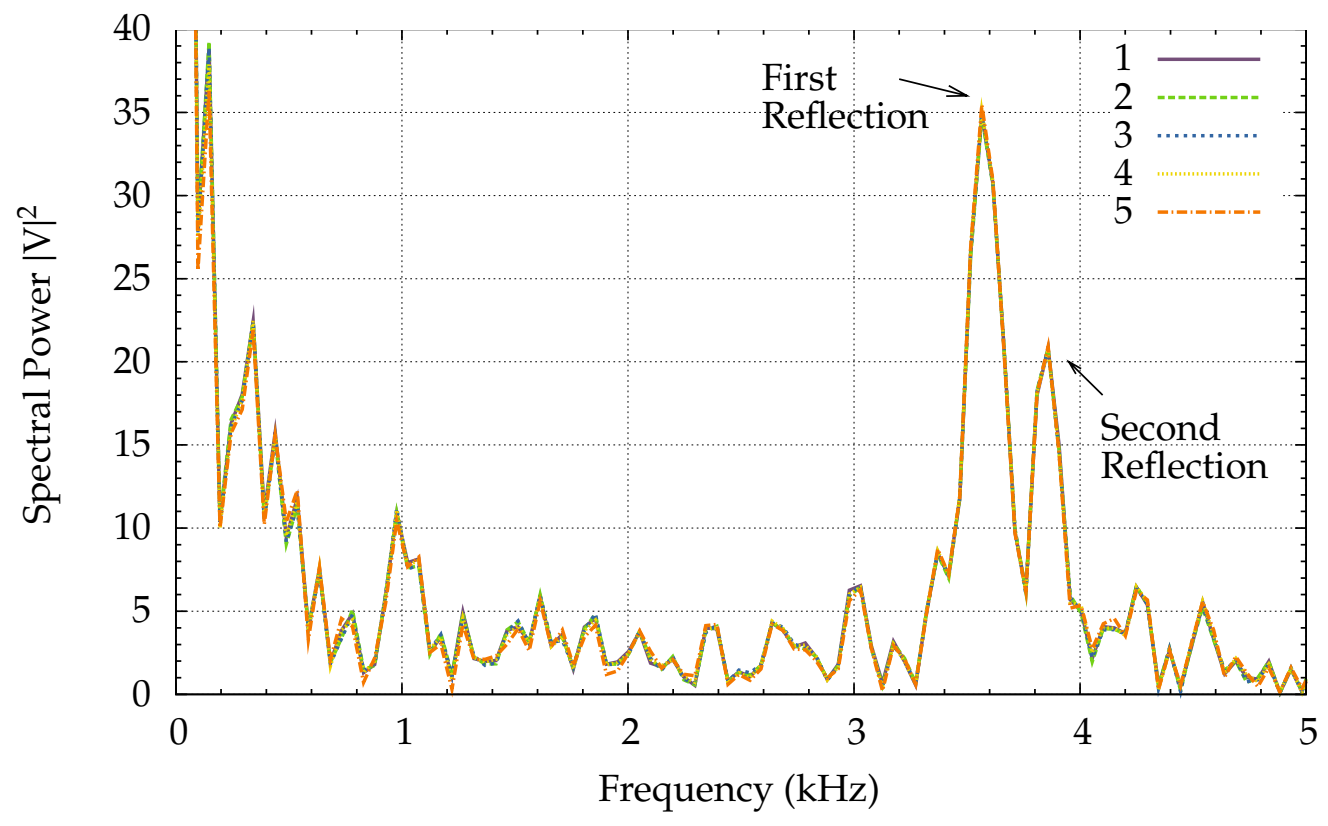

Figure 4.22: Spectrum for two objects spaced apart by $25.4 \mathrm{~cm}$. 


\section{Conclusion}

An UWB-compatible FMCW based approach can be used for boundary detection in an eldercare monitoring system. Using FMCW for determining distances provides for an elegant system architecture. This information when combined with a positioning system allows for boundary detection. With distance and the addition of an angle measurement, a position in two-dimensional space can be determined. The system can determine with reasonable accuracy the position of critical points, such corners in a room to determine the location of walls. In a cluttered environment, by using a unique passive reflector, the system is able to create a boundary in space used in a fall detection system for localization purposes. This reflector allows for uniquely identifying the systems measurement tool from background clutter. The development of a model allowed for testing system parameters. The functionality of each basic requirement was verified through those simulations and in laboratory experiments. Further testing is needed to study the robustness of this system in a real home environment where unforeseen issues can arise. 


\subsection{Future Work}

For this boundary detection system to be complete, further work is needed. A few areas to consider are discussed in the following sections.

\section{Distance Accuracy}

This thesis did not provide a result for distance accuracy. The slope error was determined to be $10 \mathrm{~cm}$. A test fixture should be developed with a more accurate measurement system to allow for precise data. In addition, horn antennas are very directional, thus precisely targeting the reflector is also a time consuming and difficult process. The reflector is not a point in space, but rather a two-dimensional sheet, and depending on the exact location where the distance measurement is referenced to, it can change the accuracy of manual measurements. Due to these tolerances, a test fixture could solve these issues and lead to more precise results for accuracy comparisons.

\section{Antennas}

One of the requirements for a monitoring system is being unobtrusive. The current horn antennas used are large, and if they were installed in a visible manner, then the system is considered obtrusive. During the experiment, the horn antennas directionality were used to aim the signal onto the reflector, so that the exact path traversed by the wavefront is known. It also allowed minimization of the effect of background "clutter". A study on the feasibility of using smaller antennas is needed to allow the final system to be considered as unobtrusive. An omni-directional antenna also needs to be considered to eliminate aiming. This allows for the reflector to be located at any location within range. 


\section{System Calibration}

A calibration of the system is required to define the measurement of zero length. The measurement of distance is based upon the frequency difference, which includes the physical distance of the signal path from the directional coupler, antennas, and LNA. This distance creates a frequency offset which is present in all points measured. Analysis should be performed on the best method to find the zero length measurement. The simplest way is to measure the frequency different with zero distance, but you can also perform the measurement at various distances and apply the regression model to estimate the value. Another consideration required is how stable is the calibration, such as over time. The effect of other environmental factors such as temperature should also be analyzed.

\section{System and Setup Costs}

The FMCW system consists mainly of semiconductor components, which are physically small. At the current state, the whole circuit of the directional coupler, mixer, and LNA fits on a small board. The current unknown is any digital section, antennas and the FMCW source (network analyzer). Limited physical size minimizes the installation costs and difficulty.

The cost for the components used is $\$ 190$. Most of the costs incurred were due to the chosen package type or the cost of sample test boards. Without these costs, the estimated cost would be lowered by at least $\$ 90$. The costs could be further reduced by re-evaluating the selected components based on required performance. When purchasing in high volume quantities, prices are reduced further. 


\section{PCB Integration}

The hardware used for the prototype was selected individually, rather than acquiring an off-the-shelf solution. This saves costs, allows for customization but increases development time. A PCB should be built to help control the circuit stability, since RF components are sensitive, this helps to minimize issues with connections and some variations during testing. Layout for ultra-wideband circuits requires high frequency layout techniques.

\section{Challenges of Integrating with An Eldercare Monitoring System}

With further analysis, this system may be integrated with the complete eldercare monitoring system. The system may use a different UWB technique for its monitoring application. With varying architectures, it could be more feasible to implement a different technique for a goal of a unified system architecture or reusing UWB circuits.

If implemented the unobtrusive monitoring system allows aiding caretakers to monitor elderly patients such as in a nursing home. In the future, the system can allow for prolonging the ability for independent living. With ongoing research into UWB, further applications and improvement in monitoring vital signs can improve such a system. 


\section{Bibliography}

[1] T. Smilkstein, "Short range indoor radar monitoring for extending independent living." [Online]. Available: http://web.missouri.edu/ smilksteint/ RadarMonitoring.html

[2] “Title 47 CRF part 15," FCC, 2009. [Online]. Available: http://www.access.gpo. gov/nara/cfr/waisidx_09/47cfr15_09.html

[3] X. Chen, Z.He, D. Anderson, J. Keller, M.Skubic, and Z. He, "Adaptive silhouette extraction and human tracking in dynamic environments 1," 2006.

[4] R. Glaser, C. Lauterbach, D. Savio, M. Schnell, S. Karadal, W. Weber, S. Kornely, and A. Stöhr, "Smart carpet: A textile-based large-area sensor network."

[5] K. Ota, Y. Ota, M. Otsu, and A. Kajiwara, “Elderly-care motion sensor using UWB-IR," in Sensors Applications Symposium (SAS), 2011 IEEE, feb. 2011, pp. $159-162$.

[6] D. Arnall. (2007, Jun.) Role reversal: The high cost of elder care. ABC News Business Unit. [Online]. Available: http://abcnews.go.com/Business/LifeStages/ story $? \mathrm{id}=3307546 \&$ page $=1$ 
[7] "Life alert," Life Alert Emergency Response, Inc. [Online]. Available: http://www.emergency.com

[8] J. S. Enoch. (2007, Sep.) Alternatives to life alert. [Online]. Available: http://www.consumeraffairs.com/news04/2007/09/lifealert_alternatives.html

[9] "Get freedom alert." [Online]. Available: http://www.getfreedomalert.com

[10] "Medical alert." [Online]. Available: http://www.callforassistance.com

[11] A. Yarovoy, L. Ligthart, J. Matuzas, and B. Levitas, “UWB radar for human being detection," Aerospace and Electronic Systems Magazine, IEEE, vol. 21, no. 11, pp. 22-26, Nov. 2006.

[12] J. C. Y. Lai, Y. Xu, E. Gunawan, E. C.-P. Chua, A. Maskooki, Y. L. Guan, K.S. Low, C. B. Soh, and C.-L. Poh, "Wireless sensing of human respiratory parameters by low-power ultrawideband impulse radio radar," Instrumentation and Measurement, IEEE Transactions on, vol. PP, no. 99, pp. 1-11, feb 2010.

[13] D. Zito, D. Pepe, M. Mincica, F. Zito, D. De Rossi, A. Lanata, E. P. Scilingo, and A. Tognetti, "Wearable system-on-a-chip UWB radar for contact-less cardiopulmonary monitoring: Present status," Aug. 2008, pp. 5274-5277.

[14] J. Bryan and Y. Kim, "Classification of human activities on UWB radar using a support vector machine," in Antennas and Propagation Society International Symposium (APSURSI), 2010 IEEE, Jul. 2010, pp. 1 -4.

[15] X. Zhuge, T. Savelyev, A. Yarovoy, L. Ligthart, J. Matuzas, and B. Levitas, "Human body imaging by microwave UWB radar," in Radar Conference, 2008. EuRAD 2008. European, Oct. 2008, pp. 148-151. 
[16] E. Staderini, "UWB radars in medicine," Aerospace and Electronic Systems Magazine, IEEE, vol. 17, no. 1, pp. 13-18, Jan. 2002.

[17] E. Taoufik, S. Nabila, and B. Ridha, "The reflection of electromagnetic field by body tissue in the UWB frequency range," May 2010, pp. 1403 -1407.

[18] E. Pancera, X. Li, L. Zwirello, and T. Zwick, "Performance of ultra wideband antennas for monitoring water accumulation in human bodies," Apr. 2010, pp. $1-5$.

[19] S. Davis, B. Van Veen, S. Hagness, and F. Kelcz, "Breast tumor characterization based on ultrawideband microwave backscatter," Biomedical Engineering, IEEE Transactions on, vol. 55, no. 1, pp. $237-246$, Jan. 2008.

[20] S. Chang, M. Wolf, and J. W. Burdick, "Human detection and tracking via ultra-wideband (UWB) radar," May 2010, pp. 452 -457.

[21] B. Allen, M. Dohler, E. E. Okon, W. Q. Malik, A. K. Brown, and D. J. Edwards, Eds., Ultra-wideband Antennas and Propagation for Communications, Radar and Imaging. John Wiely \& Sons, Ltd, 2007.

[22] H. Arslan, Z. N. Chen, and M.-G. D. Benedetto, Eds., Ultra Wideband Wireless Communication. John Wiely \& Sons, Ltd, 2006.

[23] S. Ghorashi, B. Allen, M. Ghavami, and A. Aghvami, "An overview of MB-UWB OFDM," in Ultra Wideband Communications Technologies and System Design, 2004. IEE Seminar on, Aug. 2004, pp. $107-110$.

[24] A. Batra, J. Balakrishnan, and A. Dabak, "Multi-band OFDM: a new approach for UWB," in Circuits and Systems, 2004. ISCAS '04. Proceedings of the 2004 International Symposium on, vol. 5, May 2004, pp. V-365 - V-368 Vol.5. 
[25] LLNL engineering uwb communication. [Online]. Available: https: //www-eng.llnl.gov/uwb_comm/uwb_comm.html

[26] S. Gezici, Z. Tian, G. Giannakis, H. Kobayashi, A. Molisch, H. Poor, and Z. Sahinoglu, "Localization via ultra-wideband radios: a look at positioning aspects for future sensor networks," Signal Processing Magazine, IEEE, vol. 22, no. 4, pp. $70-84$, Jul. 2005.

[27] S. Galler, W. Gerok, J. Schroeder, K. Kyamakya, and T. Kaiser, “Combined AOA/TOA UWB localization," Oct. 2007, pp. 1049 -1053.

[28] H. G. Schantz, "Smart antennas for spatial RAKE UWB systems," in Antennas and Propagation Society International Symposium, 2004. IEEE, vol. 3, Jun. 2004, pp. $2524-2527$.

[29] (2010, Jun.) Invention and initial development of monopulse radar. IEEE Global History Network. [Online]. Available: http://www.ieeeghn.org/wiki/ index.php/Invention_and_Initial_Development_of_Monopulse_Radar

[30] X. Sun, Y. Ma, J. Xu, J. Zhang, and J. Wang, “A high accuracy mono-station UWB positioning system," vol. 1, Sep. 2008, pp. $201-204$.

[31] B. Waldmann, R. Weigel, and P. Gulden, "Method for high precision local positioning radar using an ultra wideband technique," in Microwave Symposium Digest, 2008 IEEE MTT-S International, Jun. 2008, pp. 117-120.

[32] "8720C microwave network analyzer," Agilent Technologies. [Online]. Available: http://www.home.agilent.com/agilent/product.jspx?id=1000002254\% 3Aepsg\%3Apro\&pageMode=OV\&pid=1000002254\%3Aepsg\%3Apro\&lc= eng\&ct=PRODUCT\&cc=US\&pselect=SR.PM-Search\%20Results.Overview 
[33] "High power bi-directional coupler SYBD-16-53HP+ data sheet," Minicircuits. [Online]. Available: http://www.minicircuits.com/pdfs/SYBD-16-53HP+.pdf

[34] "Standard gain horns data sheet," Narda Microwave-East. [Online]. Available: http://www.nardamicrowave.com/east/index.php?m=Products\&e= details\&id=1237

[35] $50 \mathrm{ohm}$ coaxial cable - various types. PCS Electronics. [Online]. Available: http://www.pcs-electronics.com/coaxial-cable-various-types-p-357.html

[36] "Mga-86576 data sheet, 1.5 - $8 \mathrm{GHz}$ low noise," Avago. [Online]. Available: http://www.avagotech.com/docs/AV02-0608EN 


\section{Code}

The following appendix contains a selection of code use to generate data for analysis purposes.

\section{A.1 Matlab}

Matlab code used in conjunction with Simulink for preforming simulations.

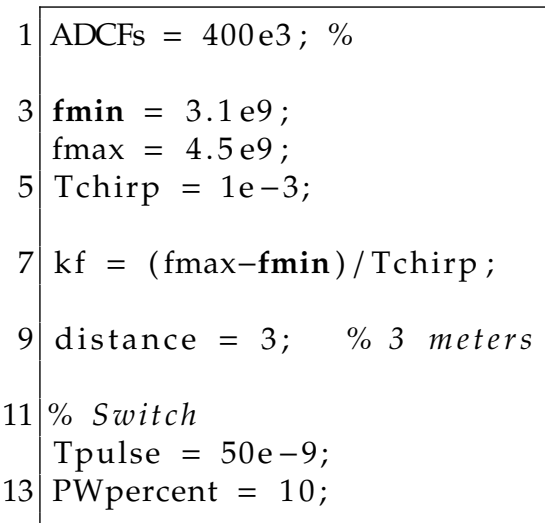

appendix/code/system_parameters.m

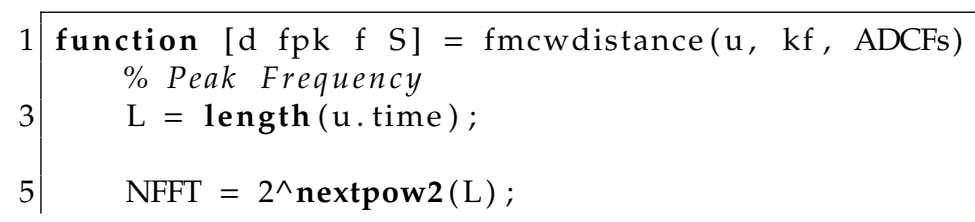




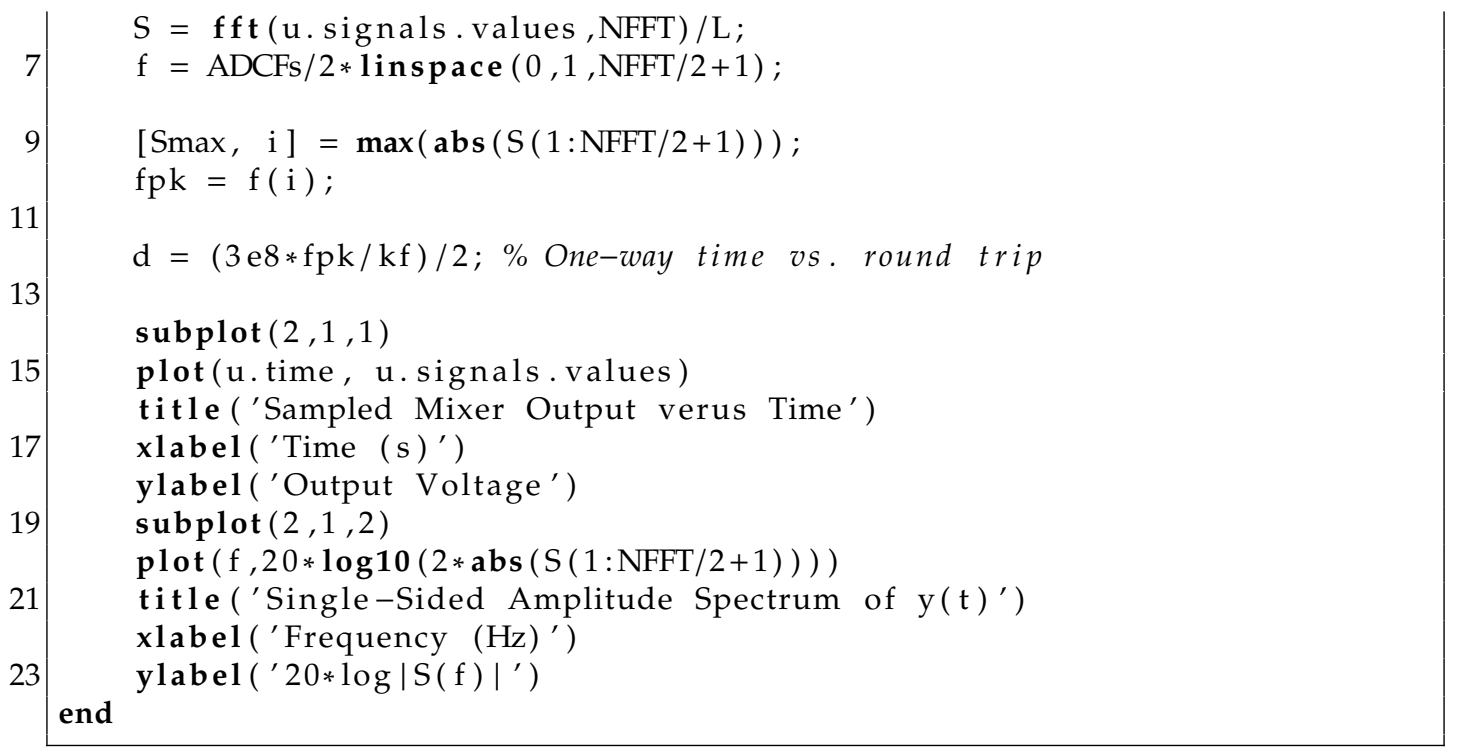

appendix/code/fmcwdistance.m

Matlab code for preforming a FFT operation.

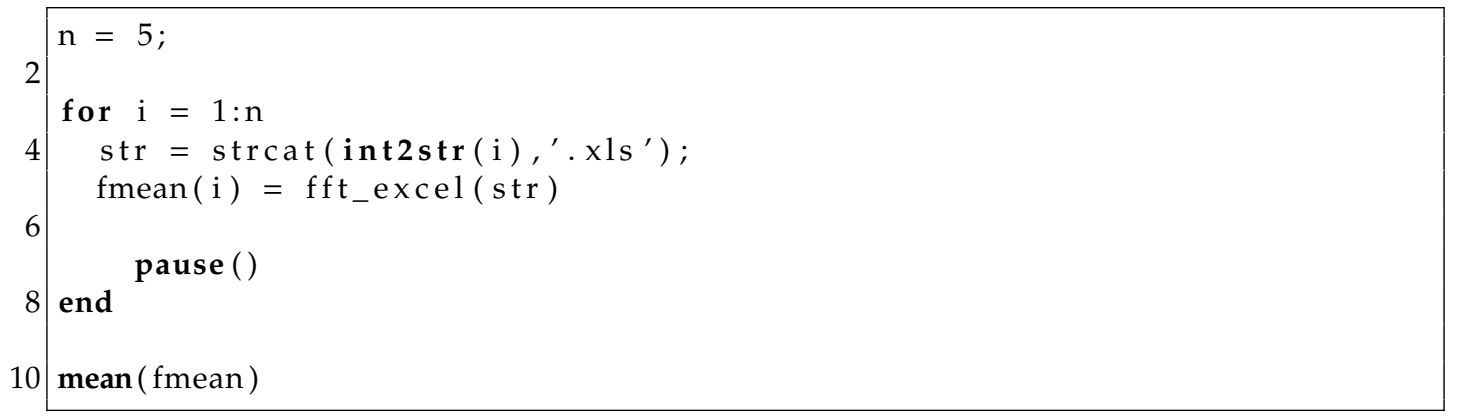

\section{fmcw/images/fft/120cm/fpeaks.m}

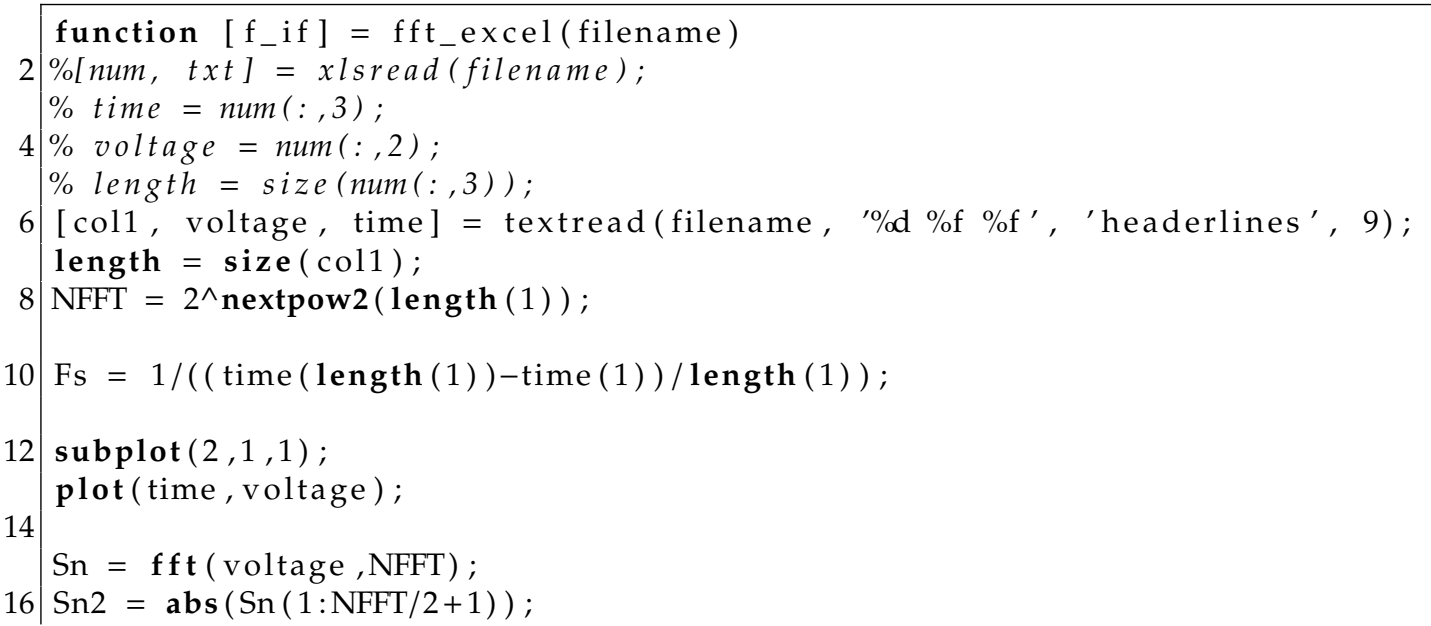




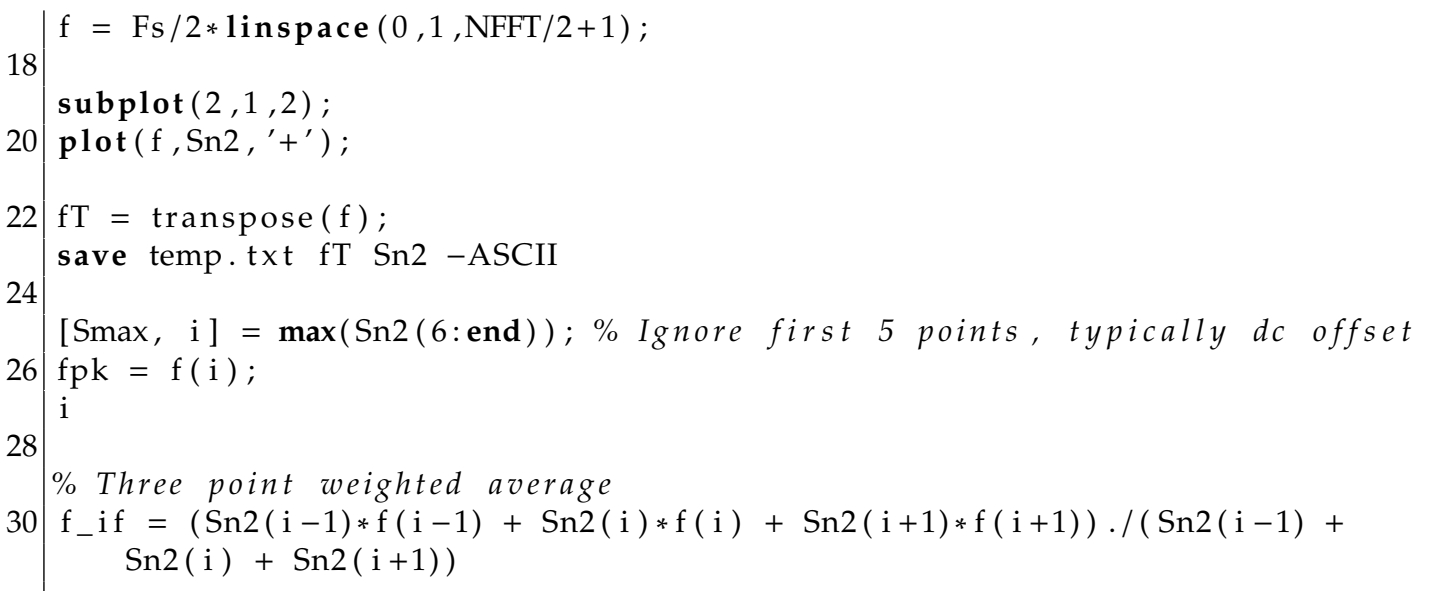

fmcw/images/fft/120cm/fft_excel.m 


\section{A.2 Gnuplot}

Gnuplot was used to create the data plots in this thesis, and to preform the ordinary least squares regression automatically. Code from a sample file and corresponding plot is shown below.

\#! / usr/bin/gnuplot

2 set terminal epslatex

set output "fmcw/images/cables-lna-data.tex"

4 set grid

set key top left

6 set size 0.9

set mxtics

set mytics

set xlabel "Distance (m)"

10 set ylabel "Frequency $(\mathrm{Hz}) "$

$f(x)=m * x+b$

12

set label 1 sprintf $(" \mathrm{f}(\mathrm{d})=\% 3.2 \mathrm{fd}+\% 3.2 \mathrm{f} ", \mathrm{~m}, \mathrm{~b})$ at $2.5,4700$

14 plot "fmcw/images/cables-lna-data.txt" using 1:2 title "measured" with points lw 4 ps 2,1

$\mathrm{f}(\mathrm{x})$ title "OLS" with lines lw 4

fmcw/images/cables-lna-data.gnu

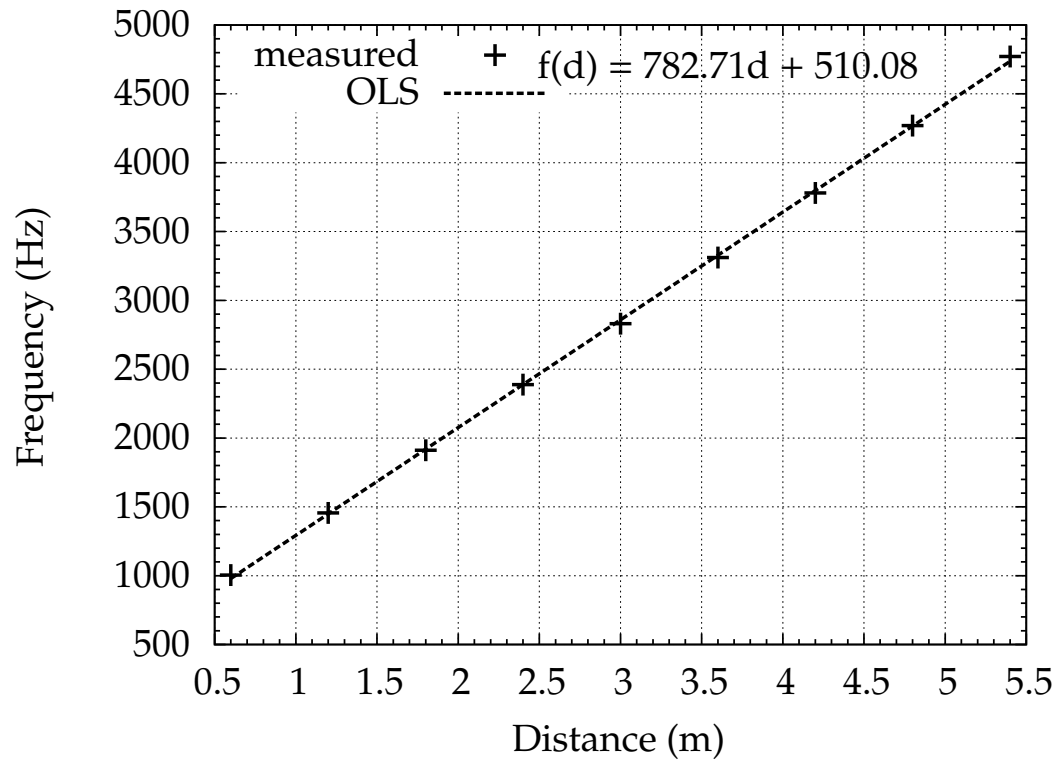


The bill of materials is located in Table B.1 with links to the corresponding distributor or manufactures datasheets.

The prices and cost are estimates for development. For implementation on a PCB, many costly test boards or packages can be eliminated such that the devices are directly soldered to the board. Additional costs for the PCB itself will be incurred. 


\begin{tabular}{|c|c|c|c|c|c|c|c|}
\hline Part No. & Distributor & Descprtion & Manufacture & Qty & Price Ea. & Price & Comments \\
\hline ZX05-C60MH-S+ & & Mixer 1600 - $6000 \mathrm{MHz} \mathrm{LO}$ & Minicircuits & 1 & 39.95 & 39.95 & \\
\hline M3SW-2-50DR+ & & $\begin{array}{l}\text { Switch, DC to } 4500 \mathrm{MHz} \text {, } \\
\text { on/off } 10 \mathrm{~ns}\end{array}$ & Minicircuits & 1 & 4.95 & 4.95 & $\begin{array}{l}\text { Surface Mount (see Test } \\
\text { Board) }\end{array}$ \\
\hline TB-159 & & Switch Test Board & Minicircuits & 1 & 44.95 & 44.95 & 6 SMA Connectors Included \\
\hline SYBD-16-53HP+ & & $\begin{array}{l}\text { Bi-directional Coupler, } 2.7 \text { - } 5 \\
\mathrm{GHz}, 16 \mathrm{~dB}\end{array}$ & Minicircuits & 1 & 29.95 & 29.95 & $\begin{array}{l}\text { Surface Mount, test board } \\
\text { available (below) }\end{array}$ \\
\hline TB-398 & & & Minicircuits & 1 & 49.95 & 49.95 & $\begin{array}{l}\text { Test board costs more than } \\
\text { the part }(6 \mathrm{sma})\end{array}$ \\
\hline MGA-86576 & \multirow[t]{2}{*}{ Digikey } & LNA $1.5^{\sim} 8 \mathrm{GHz}, 23 \mathrm{~dB} \max$ & Avago & 5 & 0 & 0 & samples 2-3 day shipping \\
\hline \multirow{2}{*}{$\begin{array}{l}\text { DEMO-MGA8657- } \\
6\end{array}$} & & Demo board (no parts) & Avago & 2 & 0 & 0 & sample \\
\hline & Haltech & $\begin{array}{l}\text { SMA Female Board Edge } \\
0.031 "\end{array}$ & & 10 & 0.95 & 9.5 & two per LNA demo board \\
\hline 490-4884-1-ND & Digikey & $\begin{array}{l}\text { Capacitor High-Q RF } 10 \mathrm{pF} \\
0805\end{array}$ & & 10 & 0.735 & 7.35 & Min buy 10 at 0.735 each \\
\hline 478-1324-1-ND & Digikey & $\begin{array}{l}\text { Capacitor General Purpose } \\
50 \mathrm{~V} 0805\end{array}$ & & 10 & 0.088 & 0.88 & Min buy 10 at 0.088 each \\
\hline \multirow[t]{2}{*}{$\begin{array}{l}\text { RMCF1/1010JRCT- } \\
\text { ND }\end{array}$} & Digikey & $\begin{array}{l}\text { Resistor } 10 \text { ohm 1\% 1/8 W } \\
0805\end{array}$ & & 10 & 0.088 & 0.88 & Min buy 10 at 0.088 each \\
\hline & & & & & Total & 188.36 & \\
\hline
\end{tabular}

Table B.1: Bill of materials 


\section{Test Procedure}

In this section, general procedures for the measurements preformed for this thesis are provided. The procedure cover characterizing the microwave devices using $S$-parameter measurements on the network analyzer, and preforming distance measurements.

\section{C.1 S-parameters}

$S$-parameter measurements are used to characterize the performance of RF devices. In general insertion loss, gain, and VSWR over frequency are variables of interest. Values can be compared with the corresponding datasheet (see Appendix B for links in the bill of materials). Past measurement data is displayed in Section 4.1 .

\section{Equipment}

- HP 8720C Network Analyzer with GPIB setup (located in anechoic chamber lab)

- RG-142 Coaxial Cable (located in microwave lab)

- Calibration kit 
- Many SMA-Female thru-lines

- Many SMA-Female to BNC-Female converters

- Banana to Grabbers (for LNA DC power supply connection)

\section{Procedure}

1. Connect RG-142 cables to port 1 and port 2 of the network analyzer.

2. Setup the frequency span: The recommended settings is for testing the system components are

- Under Stimulus, press START, enter in [ $1 \mathrm{GHz}$ ]

- Under Stimulus, press STOP, enter in [ $6 \mathrm{GHz}$ ]

3. Setup the number of points: Under Stimulus, press MENU, select NUMBER OF POINTS, select 801

4. Preform two-port calibration: Under Response, press the CAL, select CALIBRATE MENU, select RESPONSE, select FULL 2-PORT. Connect the corresponding short, open, load, and thru-line along, then press to corresponding soft key to preform the measurement.

5. Under Active Channel, press $\mathrm{CH} 1$. Under Response, press DISPLAY

6. Under Response, press FORMAT, select LOG MAG

7. Under Active Channel, press MEAS, select S11.

8. With the computer LabView program, download the S-parameter data from the network analyzer.

9. Repeat for the other S-parameters: S21, S12, S22. 


\section{C.2 Distance Measurements}

This is the setup is procedure for constructing the FMCW system to take measurement data of distances in air and coaxial cables. Pictures of the test setup are located in Section 4.2, and data results are in Section 4.3 .

\section{Equipment}

- HP 8720C Network Analyzer (located in anechoic chamber lab)

- RG-142 Coaxial Cable (located in microwave lab)

- Agilent DSO3000 series oscilloscope with USB connection and Agilent DSO3000 Scope Connect for Windows (or GPIB based setup)

- FMCW system components in Fig. C.1

- Many SMA-Female thru-lines

- Many SMA-Female to BNC-Female converters

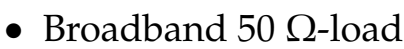

- $\mathrm{BNC}$ to BNC cables

- Banana to Grabbers (for LNA DC power supply connection)

\section{Procedure}

1. Setup the FMCW system according to Fig. C.1:

- Connect the directional coupler's forward coupling port to the LO port of the mixer

- Connect a $50 \Omega$-load to the reverse coupling port to prevent reflections 
- Secure the directional coupler and mixer, such as taping it to a board or the lab bench

- Connect the mixer's IF port to the low-pass filter

- Connect the low-pass filter output to the oscilloscope

- Connect the horn antenna to the output of the directional coupler

- Connect two LNA's together for a two-stage amplifier

- Connect the horn antenna to the LNA

- Connect the LNA output to the mixer's RF port

2. Setup the network analyzer with the following settings:

- Under Stimulus, press START, enter in [ $3 \mathrm{GHz}$ ]

- Under Stimulus, press STOP, enter in [ $4 \mathrm{GHz}$ ]

- Under Response, press AVG, select IF BW, turn the knob until $3000 \mathrm{~Hz}$ is selected

- Under Stimulus, press MENU, select MANUAL for SWEEP TIME, and enter in [ $6 \mathrm{~ms}$ ]

- For NUMBER OF POINTS, select [ 3 ]

- For POWER, enter in [ $-5 \mathrm{dBm}]$ in the corresponding range setting

This will allow the network analyzer to have the smallest sweep time

3. Trigger settings

- Setup a function generator to output a PULSED WAVEFORM at [ $50 \mathrm{~Hz}$ ]. Slow frequency is relative to the sweep time of the network analyzer. The trigger rate is about $1 / 3$ the sweep rate. The duty cycle can set to [ $5 \%$ ] 
- The output voltage of the pulse train is from $0 \mathrm{~V}$ to $2 \mathrm{~V}$

- Using a BNC-T, connect the output of the function generator to the external trigger of the oscilloscope and the network analyzer.

- Set the triggering mode on the oscilloscope to use the external trigger. Select falling edge mode for the trigger.

- The network analyzer, under Stimulus, press MENU, select TRIGGER MENU. Select SINGLE and turn on the external trigger TRIGGER: TRIG ON, and select EXT TRIG ON SWEEP for the function generator to start the sweep on the network analyzer. Note that a sweep occurs from a high to low transition of the signal.

\section{Connect a 5 V DC supply to the LNA's}

5. Point the antenna's together to test if any signal appears at the oscilloscope, if no output appears, check all settings and the circuit.

6. Preform direct distance measurements, or measurements with a reflection. If cable length measurements are desired, the amplifiers may not be required depending on the length of the cables. Do not over drive the amplifiers, the cables only loose about $1 \mathrm{~dB}$ per cable (cable length approximately $60 \mathrm{~cm}$ ). Disconnect the cables going to the antenna and connect them to each other with a thru-line.

7. Use a USB cable to connect to the Agilent DSO3000 series oscilloscope and start the corresponding program on the Windows PC. Capture the data and export the data to excel format. (Note, even though the Agilent software claims that the file is in an excel binary format, it actually saves the data as a tab delimited data file with a xls file extension, this explains any errors the user will run into when opening or saving the file in excel (or other spreadsheet programs).) 
8. Retake the data four additional times, make sure the data is triggered. If using SINGLE trigger, remember to re-trigger for new data.

9. Run the Matlab files fpeaks.m. The recommended way is to put the files fpeaks.m and fft_excel.m in the users Matlab path, typically located for Windows users in /My Doucments/MATLAB/. Run the fpeaks.m script by entering in

$$
>\text { fpeaks }
$$

in the console window. The console output will report the frequency of the recorded data, and also show the plot of the data in the time and frequency domains. 


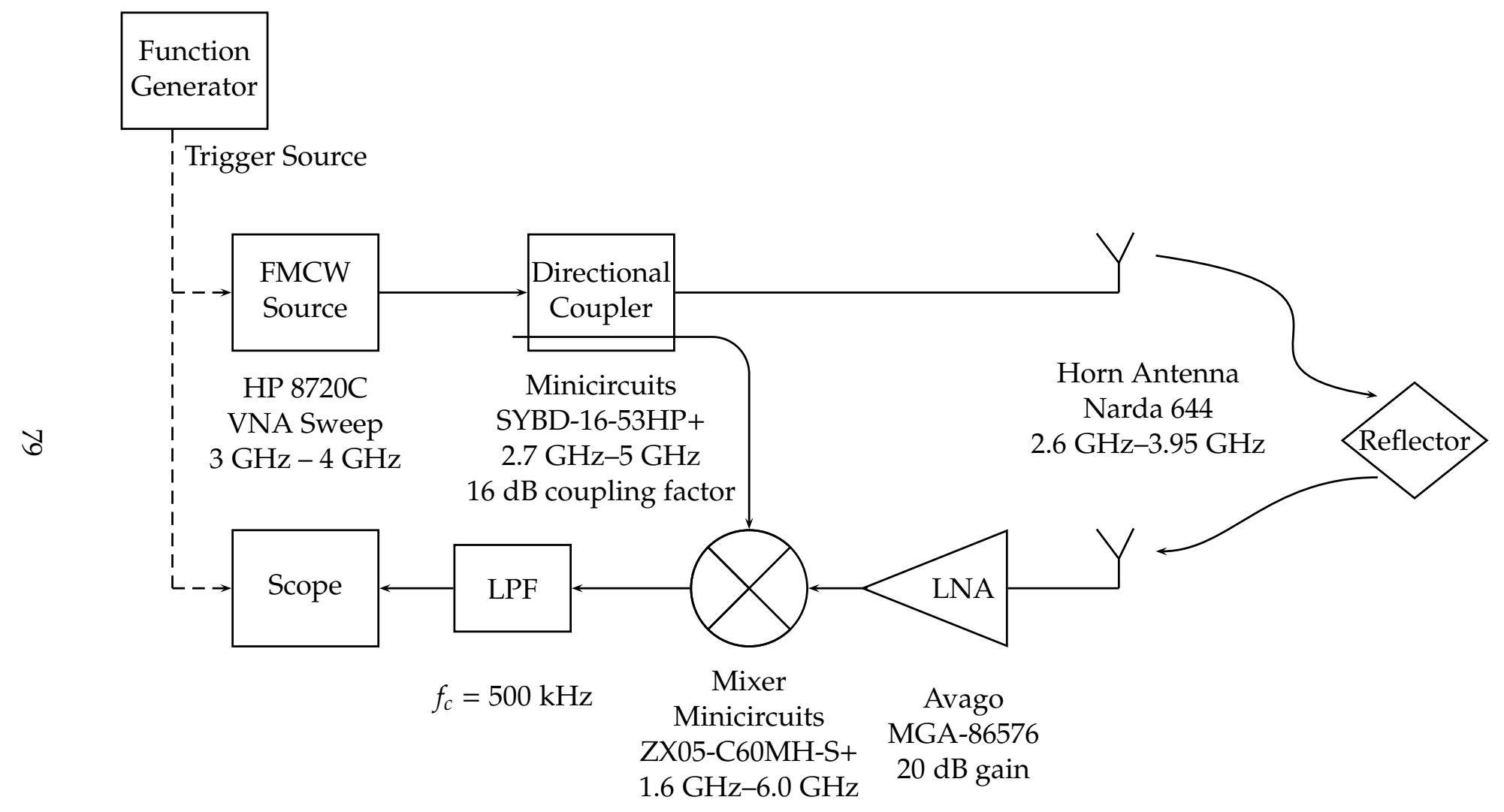

Figure C.1: Block diagram for system test setup 